\title{
A New Variational Principle and Supercritical Semilinear Elliptic Problems
}

\author{
by \\ Maryam Basiri, B.Sc. \\ A thesis submitted to \\ the Faculty of Graduate and Postdoctoral Affairs \\ in partial fulfillment of the requirements for the degree of \\ Master of Science \\ in \\ Mathematics \\ School of Mathematics and Statistics \\ Ottawa-Carleton Institute for Mathematics and Statistics \\ Carleton University \\ Ottawa, Ontario, Canada \\ (C)Copyright \\ Maryam Basiri, 2017
}




\section{Abstract}

Our objective in this thesis is to study semilinear elliptic equations of the form

$$
\left\{\begin{aligned}
-\Delta u+\lambda u & =\mu g(x, u)+b(x) & & \text { in } \Omega \\
u & =0 & & \text { on } \partial \Omega
\end{aligned}\right.
$$

where the nonlinearity $g$ may have supercritical Sobolev growth. We shall apply a new variational principle recently introduced in $[25,26]$ to prove the existence of solutions for such problems. To be more specific, assuming $G(x, \cdot)$ is the primitive of $g(x, \cdot)$ and $G^{*}(x, \cdot)$ is the Fenchel dual of $G(x, \cdot)$, we shall find a minimum of the functional $I[\cdot]$ defined by

$$
I[u]=\int_{\Omega} \mu G^{*}\left(x, \frac{-\Delta u+\lambda u-b(x)}{\mu}\right) d x-\int_{\Omega} \mu G(x, u)+b(x) u d x,
$$

over an appropriate convex set $K$, consisting of bounded functions in a certain Sobolev space. We would like to emphasize that functionals of the type (2) are different from the standard Euler-Lagrange functionals that are mostly used in the literature. The results in this thesis are twofold. Namely, we first prove the existence of a solution for (1) in the presence of a subsolution and a superso-

lution. Secondly, we consider the general case of (1), and discuss the existence and smoothness of its solutions in a supercritcal case. We remark that the latter results are recently published in [9]. 


\section{Acknowledgements}

I would like to express my deepest gratitude to my supervisor, Dr. Abbas Momeni, and thank him for his constant support, endless patience and valuable guidance throughout my master studies.

I would also like to thank him for sharing his excellent ideas based on which I could publish my first paper.

Furthermore, I extend my appreciation to the School of Mathematics and Statistics at Carleton University for accommodating me over the last two years.

I am also grateful to my parents for their enduring belief in me and their continuous encouragement.

Finally, I wish to thank my husband, Alireza, for his unconditional support without which it would be impossible for me to be where I am today. 


\section{Contents}

Title Page $\quad$ i

Abstract

Acknowledgements

Table of Contents

1 Introduction $\quad 1$

2 Preliminaries $\quad 9$

2.1 Semilinear Elliptic Partial Differential Equations . . . . . . . . . . 9

2.1.1 Linear Elliptic PDEs of Second Order . . . . . . . . . . . . 11

2.2 Functional Analysis and Weak Solutions of PDEs . . . . . . . . . 12

2.2.1 Function Spaces . . . . . . . . . . . . . . 15

2.2.2 Solutions of a Partial Differential Equation . . . . . . . . . 17

2.3 Inequalities in Sobolev Spaces . . . . . . . . . . . . . . . . . 18

2.3.1 Standard Sobolev Embeddings . . . . . . . . . . . . . . 18

2.3.2 Sobolev Embeddings for Functions of Power Forms . . . . 21

2.4 Elliptic Regularity Theory . . . . . . . . . . . . . . . . . . . . 32

2.5 Sub- and Supersolution . . . . . . . . . . . . . . . 33

2.6 Weak Maximum principle . . . . . . . . . . . . . . . . 34

2.7 Convex Analysis . . . . . . . . . . . . . . . . . . . 35 
3 Variational Problems 39

3.1 A New Variational Principle . . . . . . . . . . . . . . . . 42

3.2 Applying the New Variational Principle . . . . . . . . . . . . . . 43

4 Sub- and Supersolutions $\quad 49$

4.1 Introduction . . . . . . . . . . . . . . . . . . . . 49

4.2 Main Result . . . . . . . . . . . . . . . . 52

5 Supercritical Semilinear Elliptic Equations $\quad 59$

5.1 Introduction . . . . . . . . . . . . . . . . . . 59

5.2 Main Result . . . . . . . . . . . . . . . . 62

5.2.1 A Special Case . . . . . . . . . . . . . . 69

5.2.2 Smooth Solutions . . . . . . . . . . . . 70

$\begin{array}{ll}\text { Bibliography } & 76\end{array}$ 


\section{Chapter 1}

\section{Introduction}

In this thesis, we are interested in showing the existence of a solution for the boundary value problem of the form

$$
\left\{\begin{aligned}
-\Delta u+\lambda u & =\mu g(x, u)+b(x) & & \text { in } \Omega, \\
u & =0 & & \text { on } \partial \Omega .
\end{aligned}\right.
$$

where $\Omega$ is a bounded domain in $\mathbb{R}^{n}, \mu$ and $\lambda$ are two real parameters, $g: \Omega \times \mathbb{R} \rightarrow$ $\mathbb{R}$ is a Carátheodory function, which means $g(\cdot, u)$ is measurable for all $u \in \mathbb{R}$, and $g(x, \cdot)$ is continuous a.e. in $\Omega$, and $b: \Omega \rightarrow \mathbb{R}$ is a function belonging to an appropriate space. There have been numerous studies about elliptic equations such as (1.1), and to prove the existence of solutions for this problem either topological methods, such as fixed point and sub- and supersolution method, or variational tools have been used in the literature.

In this thesis we prove the existence of solutions for (1.1) via a new variational principle introduced in [26]. Generally, in a variational principle we are working on a specific kind of problems for which we can find an energy functional $\Phi$, defined on an appropriate function space, whose critical points are in one-to-one correspondence with solutions of the partial differential equation (PDE). With this approach we can avoid facing various complications of finding solutions for (1.1), and take advantage of several well-known theorems and results about the 
existence of critical points of a functional, such as constrained minimization or mountain pass method, in the process of the proof of our desired result.

As shown in [26], there are infinitely many functionals with this desired property which can be associated with a given PDE such as (1.1). One of these functionals, which has been used frequently in the literature, is the Euler-Lagrange functional. Let $V$ be a Banach space with $V^{*}$ its topological dual and set $\langle\cdot, \cdot\rangle$ to be the duality pairing between them. We can look at PDE (1.1) abstractly as $\Lambda[u]=0$, where $\Lambda: V \rightarrow V^{*}$ is the appropriate nonlinear partial differential operator, and assume that $\Lambda[u]=0$ is the Euler-Lagrange equation for an appropriate Gâteaux differentiable functional $\Phi$. In this way we have $D \Phi[u]=\Lambda[u]$, and hence any critical point $u$ of $\Phi$, where $D \Phi[u]=0$ corresponds to a solution of the partial differential equation $\Lambda[u]=0$. For instance, consider the particular case of (1.1) as below

$$
\left\{\begin{aligned}
-\Delta u+\lambda u & =|u|^{p-2} u & & \text { in } \Omega, \\
u & =0 & & \text { on } \partial \Omega .
\end{aligned}\right.
$$

Setting $V$ to be the Sobolev space $H_{0}^{1}(\Omega)=W_{0}^{1,2}(\Omega)$, consisting of functions that together with their first weak derivatives belong to the Banach space $L^{2}(\Omega)$ and vanish on the boundary, it can be easily seen that the Euler-Lagrange functional associated to this problem is

$$
\Phi[u]=\int_{\Omega}\left(\frac{|\nabla u|^{2}}{2}+\frac{\lambda|u|^{2}}{2}-\frac{|u|^{p}}{p}\right) d x .
$$

Regardless of the approach taken for solving (1.1), it is well-known that these problems do not always admit solutions. Some assumptions on $\Omega$, such as its shape and smoothness, on $g$, including its monotonicity and growth, and also on $b$, the space to which it belongs, have to be in place in order to obtain solutions for (1.1). One of the assumptions that has a huge impact on the solvability of (1.1) is the growth of the nonlinearity $g$. For $n \geq 3$, we say that $g$ has subcritical, 
critical and supercritical growth at infinity respectively if

$$
\lim _{r \rightarrow \infty} \frac{|g(\cdot, r)|}{|r|^{2^{*}-1}}=0, \quad 0<\lim _{r \rightarrow \infty} \frac{|g(\cdot, r)|}{|r|^{2^{*}-1}}<\infty, \quad \lim _{r \rightarrow \infty} \frac{|g(\cdot, r)|}{|r|^{2^{*}-1}}=\infty,
$$

where $2^{*}=2 n /(n-2)$. Hence, for the special case (1.2), these conditions are respectively equivalent to $p<2^{*}, p=2^{*}$ and $p>2^{*}$. The important point to note here is that $2^{*}$ is the Sobolev critical exponent, in the sense that for any value $1<p<2^{*}$ the embedding $H_{0}^{1}(\Omega) \hookrightarrow L^{p}(\Omega)$ is compact.

Many works on this subject have been concerned with the subcritical problem. Using the subcriticality of the problem in addition to some other hypotheses on $g$, the existence of at least one solution for (1.1) has been proved via various methods (see [24, 39, 36, 31] for the detailed review of these approaches). For instance, applying a variational method via the Euler-Lagrange functional to the particular case (1.2), Willem in [39] showed that the problem admits at least one solution for every value of $\lambda \in \mathbb{R}$ and has a positive solution for $\lambda \geq-\lambda_{1}$, where $\lambda_{1}$ is the first eigenvalue of $-\Delta$ on $H_{0}^{1}(\Omega)$. The simplicity of the proof arises from the fact that the compactness of Sobolev embedding $H_{0}^{1}(\Omega) \hookrightarrow L^{p}(\Omega)$ implies that the functional $\Phi$ in (1.3) satisfies the Palais-Smale condition. Therefore, one can use the mountain pass theorem to show the existence of a critical point of the functional $\Phi$ as desired. It is worth noting that in the subcritical case the existence of solutions does not depend on the shape of $\Omega$.

Finding a solution for (1.1) when the nonlinearity is critical is not as straight forward. This is caused by the lack of compactness of the Sobolev embedding $H_{0}^{1}(\Omega) \hookrightarrow L^{p}(\Omega)$. This change in solvability is so dramatic that in [30] it has been shown by Pohozaev that the problem (1.2) with $\lambda \geq 0$, does not have any positive solution when $\Omega$ is starshaped and $p=2^{*}$. However, Brezis and Nirenberg proved in [10] that this situation can be rectified and a positive solution of (1.2) can be found provided that $-\lambda_{1}<\lambda<\lambda^{*}$, where $\lambda^{*}=0$ for $n \geq 4$ and $-\lambda_{1}<\lambda^{*} \leq 0$ if $n=3$. In their proof, instead of directly finding a critical point of the functional $\Phi$ defined in (1.3), equivalently they showed that if $\lambda$ takes appropriate values 
then

$$
\inf _{\substack{u \in H_{D}^{1}(\Omega) \\\|u\|_{L^{p}(\Omega)=1}}}\left\{\int_{\Omega}|\nabla u|^{2} d x-\lambda \int_{\Omega} u^{2} d x\right\},
$$

is achieved. The challenge in their approach, caused by lack of compactness, is that they do not have the continuity of the function $u \rightarrow\|u\|_{L^{p}(\Omega)}$ under weak convergence in $H_{0}^{1}(\Omega)$. Hence, the proof of the desired result is not immediate. In order to overcome this issue, they first showed that

$$
\inf _{\substack{u \in H_{0}^{1}(\Omega) \\\|u\|_{L^{p}(\Omega)=1}}}\left\{\int_{\Omega}|\nabla u|^{2} d x-\lambda \int_{\Omega} u^{2} d x\right\} \leq \inf _{\substack{u \in H_{0}^{1}(\Omega) \\\|u\|_{L^{p}(\Omega)=1}}}\left\{\int_{\Omega}|\nabla u|^{2} d x\right\},
$$

for appropriate values of $\lambda$ depending on the dimension $n$. Then, they proved for each range of $n$ that if (1.6) holds, the infimum in (1.5) is achieved. Other approaches have been taken by other authors in $[3,16,39]$ to prove the existence of solutions of (1.1) for critical case.

In supercritical case the embedding $H_{0}^{1}(\Omega) \hookrightarrow L^{p}(\Omega)$ is no longer true, so the issues arising in the process of solving problem (1.1) are more severe. For instance, in case of problem (1.2) the functional $\Phi$ defined in (1.3) is not welldefined on $H_{0}^{1}(\Omega)$ any more, so the classical variational arguments that have been applied before, are not applicable in this case. As a result, most researchers used other arguments for solving the supercritical problem. Searching for a positive radially symmetric solution $u=u(r)$, where $r=|x|$, Dolbeault and Flores in [18] considered the problem (1.2) in a ball. In this way, they were able to transform the problem into an ODE of the form

$$
\left\{\begin{array}{rr}
-u^{\prime \prime}-\frac{n-1}{r} u^{\prime}+\lambda u=u^{p-1}, & 0<r<1, \\
u^{\prime}(0)=0, & u(1)=0 .
\end{array}\right.
$$

Then, by using ODE techniques they showed that there exists a specific value of $\lambda^{*}<0$ such that for $\lambda$ near $\lambda^{*}$ the problem admits positive solutions provided that $p<(2 n-4 \sqrt{n-1}-4) /(n-2 \sqrt{n-1}-4)$ or $n \leq 10$, in other words $p$ can 
include supercritical values but it cannot be too large. In [8], Barroso considered problem (1.1) with $b(x) \in L^{2}(\Omega)$ and $\lambda$ close to zero. He utilized a fixed point approach and proved the existence of a solution of the problem (1.1) for $p>2$ if $n=3$, for $2 \leq p \leq(2 n-4) /(n-4)$ if $n>4$, and finally for $2<p \leq 3$ if $n=4$. Having an additional assumption of the existence of sub- and supersolutions for problem $(1.1)$ with $b(x)=0$, the authors in $[2,14,17,15]$ solved the problem for the supercritical case. In addition, many authors considered the effect of the topology of the domain in the solvability of problem (1.1) in the supercritical case. While in a starshaped domain, the nonexistence result of Pohozaev for problem (1.2) with $\lambda \geq 0$, is still true for $p>2^{*}$, Kazdan and Warner have shown in [23] that this problem admits solutions for any value of $p>2$ if $\Omega$ is an annulus. Also, Passaseo proved in [29] that for every $p>2^{*}$ there exists a contractible domain in which the particular case (1.2) has solutions. The dependence of solutions to the supercritical problem on other types of domains is also discussed in $[32,28]$.

In this thesis, we apply a new variational principle established in $[25,26]$ to prove the existence of solutions for supercritical problem (1.1) on a bounded domain with a smooth boundary. This principle will rectify the difficulties introduced by lack of compactness, and enables us to find solutions for our problem.

As seen in the cited literature above, the Euler-Lagrange functional cannot always be used. Here, in our new principle, we use one of the new functionals introduced in [26]. To briefly introduce the new functional, let us find it for the particular case (1.2).

Let $V=H_{0}^{1}(\Omega) \cap L^{p}(\Omega)$, and consider a closed convex subset $K$ of $V$. Let $V^{*}$ be the topological dual of $V$ and define the linear operator $A: \operatorname{Dom}(A) \subset V \rightarrow V^{*}$ by $A:=-\Delta+\lambda I$. Set $g(x, u)=|u|^{p-2} u$ and define

$$
G(x, u)=\int_{0}^{u} g(x, s) d x=\frac{1}{p}|u|^{p} .
$$


Set $\varphi: V \rightarrow \mathbb{R}$ to be

$$
\varphi(u):=\int_{\Omega} G(x, u) d x=\frac{1}{p} \int_{\Omega}|u|^{p} d x .
$$

Note that we can rewrite the problem (1.1) as

$$
A u=D \varphi(u)
$$

where $D \varphi(\cdot)$ is the Gâteaux derivative of $\varphi$. Let $G^{*}: \Omega \times \mathbb{R} \rightarrow(-\infty,+\infty]$ be the Fenchel dual of $G$, i.e.

$$
G^{*}(x, s)=\sup _{t \in \mathbb{R}}\{t s-G(x, t)\}
$$

By an easy calculation, we explicitly find $G^{*}$ as below

$$
G^{*}(x, s)=\frac{1}{p^{\prime}}|s|^{p^{\prime}}
$$

where $p^{\prime}=\frac{p}{1-p}$ is the Sobolev conjugate of $p$. As we will prove in the proceeding chapters, the Fenchel dual $\varphi^{*}: V^{*} \rightarrow \mathbb{R}$ of $\varphi$ at $A u$ is given by

$$
\varphi^{*}(A u)=\int_{\Omega} G^{*}(x, A u) d x=\frac{1}{p^{\prime}} \int_{\Omega}|-\Delta u+\lambda u|^{p^{\prime}} d x
$$

Now following [25, 26], we find the new energy functional $I[\cdot]$ over $K \cap W^{2, p^{\prime}}(\Omega)$ to be

$$
I[u]=\varphi^{*}(-\Delta u+\lambda u)-\varphi(u)=\frac{1}{p^{\prime}} \int_{\Omega}|-\Delta u+\lambda u|^{p^{\prime}} d x-\frac{1}{p} \int_{\Omega}|u|^{p} d x .
$$

The symmetric nature of this new functional, provided by the existence of a function $G$ and its Fenchel dual $G^{*}$, alleviates the difficulty and shortens the process of showing the existence of solutions for problems with supercritical nonlinearity. In order to prove the existence of a solution for (1.1), we shall show that any critical point of the functional $I[\cdot]$ on $K$ is indeed a solution of (1.1) on $V$. This is where the new variational approach will be applied. Here we state the new variational principle as introduced in [26]. 
Theorem 1.0.1. Let $V$ be a reflexive Banach space and $K$ be a closed convex subset of $V$. Let $\varphi: V \rightarrow \mathbb{R}$ be a Gâteaux differentiable convex and lower semicontinuous function, and let the linear operator $A: \operatorname{Dom}(A) \subset V \rightarrow V^{*}$ be symmetric and positive. Assume that $u$ is a critical point of $I(w)=\varphi^{*}(A w)-$ $\varphi(w)$, and that there exists $v \in K$ satisfying the linear equation,

$$
A v=D \varphi(u) .
$$

Then $u \in K$ is a solution of the equation,

$$
A u=D \varphi(u) .
$$

One of the advantages of using this new variational principle lies in the fact that instead of finding the critical point on the whole space, which is challenging when the nonlinearity of the problem is supercritical, we find it on an appropriate closed and convex set $K$. For example, for problems that we are considering in this thesis, looking for a minimizer in either a bounded set or small closed ball instead of the whole space $V$, and using the boundedness of elements of $K$, provides us with the needed compactness as we can use the compact embeddings for Sobolev spaces. Showing that the critical point of the functional in the small set corresponds to its critical point in $V$ is the next challenge. However, in the new principle this problem is reduced to just proving that the linear equation $A v=D \varphi(u)$, where $u \in K$ is given, has a solution $v \in K$. In our cases, the existence of a solution for the linear equation follows from the standard existence theory. Finally, to prove that this solution is indeed in $K$, in each case of our problems we make use of either the maximum principle or $L^{p}$ estimates and elliptic regularity theory.

The thesis is organized as follows. In Chapter 2, we review some preliminary results and basic definitions about each aspect of our work. We begin by a short introduction to semilinear elliptic partial differential equations and their solutions. Then, we compile some important facts about Sobolev spaces, and divided into a 
sequence of lemmas, we prove some interesting results as an application of Sobolev embeddings theory. We then proceed by bringing some important theorems about elliptic regularity, $L^{p}$ estimates and the maximum principle, to which we refer frequently throughout the thesis. Finally, we look into some essential results and definitions in convex analysis of lower semi-continuous functions which we need for defining the new functional.

In Chapter 3, after a brief exposition to variational problems, we present the new variational principle, which is the essence of the proof of main results in this thesis. Then, we explain the applications of the new principle for our case. Considering a less complicated version of (1.1), where $b(x)=0$, and $\lambda=$ $0, \mu=1$, in Chapter 4 we prove the existence of a solution when the supercritical problem has weak sub- and supersolutions. By assuming this extra hypothesis, we will show how the combination of the new variational principle, and suband supersolution method enables us to overcome the complication of solving the supercritical problem.

Furthermore, in Chapter 5 we work on the general case of (1.1) with supercritical nonlinearity. When $b(x) \in L^{d}(\Omega)$, with $d>2$ a real number, and $g$ satisfies an appropriate growth condition $g(x, u) \leq a|u|^{p-1}+c$ with $a, c$ constants, and $p>2$, we find a strong solution $u \in W^{2, d}(\Omega)$ for relatively good range of $p>2^{*}$. This result will be achieved by backing up the new variational principle with Sobolev compact embeddings and $L^{p}$ estimates theory for Dirichlet problems. Later in this chapter for a special case of (1.1), where $g(x, u)=|u|^{p-2} u$ and $p>2$, we will show that for a similar range of $p$ a more smooth solution can be found provided that $b(x)$ has a higher regularity. 


\section{Chapter 2}

\section{Preliminaries}

In this chapter, we review some definitions and results to which we refer frequently throughout the rest of this thesis.

The main purpose of this thesis is to show the existence of a solution for a certain type of semilinear PDEs. Hence, we start this section by reviewing some basic definitions and results in this subject.

\subsection{Semilinear Elliptic Partial Differential Equa- tions}

Consider $\Omega$ to be an open subset of $\mathbb{R}^{n}$ and $u: \Omega \rightarrow \mathbb{R}$ to be a $k$-times continuously differentiable function. Then for $\alpha=\left(\alpha_{1}, \cdots, \alpha_{n}\right)$ a multi-index, with $|\alpha|=$ $\alpha_{1}+\cdots+\alpha_{n}$ and $0 \leq \alpha_{i} \in \mathbb{Z}$ for $i=1, \cdots, n$, we define

$$
D^{\alpha} u(x):=\frac{\partial u(x)}{\partial x_{1}^{\alpha_{1}} \ldots \partial x_{n}^{\alpha_{n}}}=u_{x_{1}}^{\alpha_{1}} \ldots u_{x_{n}}^{\alpha_{n}} .
$$

It follows that for any $k$-th order partial derivative of $u(x)$, where $k$ is a positive integer, we have that $D^{k} u(x)=\left(D^{\alpha} u(x)\right)_{|\alpha|=k}$ and $\left|D^{k} u(x)\right|=\left(\sum_{|\alpha|=k}\left|D^{\alpha} u(x)\right|^{2}\right)^{\frac{1}{2}}$. A $k$-th order PDE can be represented by an equation of the form

$$
F\left(D^{k} u(x), D^{k-1} u(x), \cdots, D u(x), u(x), x\right)=0, \quad(x \in \Omega),
$$


where

$$
F: \mathbb{R}^{n^{k}} \times \mathbb{R}^{n^{k-1}} \times \cdots \times \mathbb{R}^{n} \times \mathbb{R} \times \Omega \rightarrow \mathbb{R}
$$

is given and $u: \Omega \rightarrow \mathbb{R}$ is the unknown. Ideally, one may look for a function $u$ satisfying (2.1) along with a boundary condition on $\partial \Omega$. However, it may not be possible to find such a function explicitly for all PDEs. In these cases showing the existence of a solution will be of interest.

In addition to their order, PDEs are classified in two other useful ways. One of these classifications is based on the linearity or nonlinearity of the PDE. In this work, we are focused on semilinear PDEs, which in general have the following form

$$
\sum_{|\alpha|=k} a_{\alpha}(x) D^{\alpha} u+a_{0}\left(D^{k-1} u, \cdots, D u, u, x\right)=0 .
$$

In particular when we have sufficient smoothness, a semiliear equation of second order can be written as

$$
\sum_{i, j=1}^{n} a^{i j}(x) u_{x_{i} x_{j}}+a_{0}(D u, u, x)=0 .
$$

where $A(x)=\left[a^{i j}(x)\right]$ is a symmetric matrix, i.e. $a^{i j}=a^{j i}$, and $D u=\left(u_{x_{1}}, \cdots, u_{x_{n}}\right)$.

In the other classification, where PDEs are categorized as parabolic and hyperbolic and elliptic, the arrangement is based on some qualitative properties of PDEs from which the existence and uniqueness and regularity of their solutions can be implied in the same or very similar ways for the PDEs within the same class (see [21] for more details).

In this work, we are concerned with elliptic equations. In the case of the second order semilinear equations (2.2), ellipticity of an equation can be guaranteed if the symmetric matrix $A(x)=\left[a^{i j}(x)\right]$ is positive definite.

As one can see, equation (1.1), on which we are working in this thesis, is a particular case of semilinear elliptic equations of second order with a Dirichlet boundary condition, $u=0$ on $\partial \Omega$. 
The theory of elliptic equations of second order is well-developed for linear equations (see [21],[22]). Moreover, as a part of the new principle which we are applying to problem (1.1), we need to find a solution of the associated linear equation to our problem. Hence, to approach the semilinear problem of the same type we will need the theories and results for the linear equations. We devote the next sub-section to the introduction of linear elliptic equations.

\subsubsection{Linear Elliptic PDEs of Second Order}

Here, we consider a boundary value problem of the following form

$$
\left\{\begin{array}{rll}
L u=f & \text { in } & \Omega \\
u=0 & \text { on } & \partial \Omega,
\end{array}\right.
$$

where $\Omega$ is a bounded domain in $\mathbb{R}^{n}, f: \Omega \rightarrow \mathbb{R}$ is a given function, and $L$ is a second order partial differential operator having either divergence form

$$
L u=\sum_{i, j=1}^{n}\left(a^{i j}(x) u_{x_{j}}+b^{i}(x) u\right)_{x_{i}}+\sum_{i=1}^{n} c^{i}(x) u_{x_{i}}+d(x) u,
$$

or nondivergence form

$$
L u=\sum_{i, j=1}^{n} a^{i j}(x) u_{x_{i} x_{j}}+\sum_{i=1}^{n} b^{i}(x) u_{x_{i}}+c(x) u,
$$

for given coefficient functions $a^{i j}, b^{i}, c^{i}, c, d,(i, j=1, \ldots, n)$. We also assume that the matrix $A(x)=\left[a^{i j}(x)\right]$ is symmetric, i.e. $a^{i j}=a^{j i}$ for $i, j=1, \ldots, n$. Now we bring the formal definition of elliptic equations from [21].

Definition 2.1.1 (Elliptic equations). We say the partial differential operator $L$ is (uniformly) elliptic if there exists a constant $\theta>0$ such that

$$
\sum_{i, j=1}^{n} a^{i, j}(x) \zeta_{i} \zeta_{j} \geq \theta|\zeta|^{2},
$$

for a.e. $x \in \Omega$ and all $\zeta=\left(\zeta_{1}, \cdots, \zeta_{n}\right) \in \mathbb{R}^{n}$. When $a^{i j}=\delta_{i j}, b^{i}=0$ and $c=0$, the operator $L$ is $\Delta$ (see [21, p.313]). 
One of the most common approaches for solving a partial differential equation, which we are also following in the new principle, is to look at it as an operator, say $A$, defined on appropriate linear function spaces. For the operator to be well defined, first we need to find spaces $V, W$ on which we can define $A: V \rightarrow W$. This is the point where the concept of functional analysis relates to the problem of solving a PDE. As a result, in the following section we provide some of the required materials from the theory of functional analysis.

\subsection{Functional Analysis and Weak Solutions of PDEs}

Consider a linear space $V$ over $\mathbb{R}$, and recall that a norm on $V$ is a mapping $\|\cdot\|: V \rightarrow \mathbb{R}$ which satisfies the following conditions;

(i) $\|u\| \geq 0$ for all $u \in V,\|u\|=0$ if and only if $u=0$.

(ii) $\|\alpha u\|=|\alpha|\|u\|$ for all $\alpha \in \mathbb{R}, u \in V$.

(iii) $\|u+v\| \leq\|u\|+\|v\|$ for all $u, v \in V$ (see [21, p.719] for more details).

Base on the norm on the space $V$, we can define the notion of a convergent sequence and furthermore a Cauchy sequence as follows.

Definition 2.2.1 (Strong Convergence). A sequence $\left\{u_{n}\right\} \subset V$ is said to converge strongly to $u \in V$, denoted by $u_{n} \rightarrow u$, if $\lim _{n \rightarrow \infty}\left\|u_{n}-u\right\|=0$ (see [21, p.719]).

Definition 2.2.2 (Cauchy sequence). A sequence $\left\{u_{n}\right\} \subset V$ is called a Cauchy sequence, if for each $\epsilon>0$, there exists $N>0$ such that

$$
\left\|u_{n}-u_{m}\right\|<\epsilon
$$

for all $n, m \geq N$. 
When these two notions are equivalent in a space $V$, that is every Cauchy sequence is convergent, then $V$ is called complete. Now we bring the following definitions from [21].

Definition 2.2.3 (Banach spaces). A Banach space $V$ is a complete, normed linear space.

For every Banach space we can define a dual space as follows.

Definition 2.2.4 (Dual spaces). The collection of all bounded linear functionals on $V$, that is $u^{*}: V \rightarrow \mathbb{R}$, is called the dual space of $V$ and is denoted by $V^{*}$. For $u \in V$ and $u^{*} \in V^{*}$, we denote the real number $u^{*}(u)$ by $\left\langle u^{*}, u\right\rangle$. The symbol $\langle\cdot, \cdot\rangle$ denotes the pairing between $V$ and $V^{*}$.

Definition 2.2.5. A Banach space is reflexive if $\left(V^{*}\right)^{*}=V$. This meanes that for each $u^{* *} \in\left(V^{*}\right)^{*}$, there exists $u \in V$ such that

$$
\left\langle u^{* *}, u^{*}\right\rangle=\left\langle u^{*}, u\right\rangle \quad \text { for all } u^{*} \in V^{*} \text {. }
$$

Some of the most important Banach spaces are $L^{p}$ spaces and Hilbert spaces.

Definition 2.2.6 ( $L^{p}$ Spaces). Assume $\Omega$ is an open subset of $\mathbb{R}^{n}$ and $1 \leq p \leq \infty$. For a measurable function $u: \Omega \rightarrow \mathbb{R}$, we set

$$
\|u\|_{L^{p}(\Omega)}:= \begin{cases}\left(\int_{\Omega}|u|^{p} d x\right)^{1 / p}, & \text { if } 1 \leq p<\infty, \\ {\operatorname{ess}-\sup _{\Omega}|u|,} \mid & \text { if } p=\infty\end{cases}
$$

and define $L^{p}(\Omega)$ to be the linear space of all measurable functions $u: \Omega \rightarrow \mathbb{R}$ for which $\|u\|_{L^{p}(\Omega)}$ is finite. We can also define $L_{\mathrm{loc}}^{p}(\Omega):=\left\{u ; u \in L^{p}\left(\Omega^{\prime}\right)\right.$, for all $\Omega^{\prime} \subset$ $\Omega, \Omega^{\prime}$ comact\}. (See [21, p. 702] for more details).

For $1 \leq p<\infty$ the dual space of $V=L^{p}(\Omega)$ is $V^{*}=L^{p^{\prime}}(\Omega)$, where $1 / p+1 / p^{\prime}=$ 1 and $1<p^{\prime} \leq \infty$. In addition, $L^{p}(\Omega)$ spaces are reflexive for $1<p<\infty$. 
Definition 2.2.7 (Hilbert spaces). Assume that $H$ is a linear space. A mapping $(\cdot, \cdot): H \times H \rightarrow \mathbb{R}$ is called an inner product if

(i) $(u, v)=(v, u)$ for all $u, v \in H$,

(ii) the mapping $u \rightarrow(u, v)$ is linear for each $v \in H$,

(iii) $(u, u) \geq 0$ for all $u \in H$,

(iv) $(u, u)=0$ if and only if $u=0$.

A Hilbert space is a Banach space equipped with an inner product that generates the norm. (See [21, p. 720] for more details.)

By using the notion of dual of a Banach space, another weaker concept of convergence in $V$ can be defined in the following sense.

Definition 2.2.8 (Weak convergence). A sequence $\left\{u_{n}\right\} \subset V$ is said to converge weakly to $u \in V$, denoted by $u_{n} \rightarrow u$ if

$$
\left\langle u^{*}, u_{n}\right\rangle \rightarrow\left\langle u^{*}, u\right\rangle
$$

for each functional $u^{*} \in V^{*}$.

Now, we recall the following theorems without proof.

Theorem 2.2.9 (Weak compactness). A bounded sequence in a reflexive Banach space contains a weakly convergent subsequence (see [22, p.85] for a proof).

Theorem 2.2.10. Let $\Omega$ be a measurable set, If a sequence $u_{n} \rightarrow u$ strongly in $L^{p}(\Omega)$ for $1 \leq p \leq \infty$, then a subsequence of $\left\{u_{n}\right\}$ converges to $u$ a.e. on $\Omega$ (see [33, p. 148] for a proof).

We finish this section by giving some standard definitions about linear operator on a Banach space. 
Definition 2.2.11. Let $V$ be a reflexive Banach space and $V^{*}$ its topological dual. A linear operator $A: \operatorname{Dom}(A) \subset V \rightarrow V^{*}$ is called symmetric if $\operatorname{Dom}(A)$ is dense in $V$ and $\langle A u, v\rangle=\langle u, A v\rangle$ for all $u, v \in \operatorname{Dom}(A)$. The operator $A$ is said to be positive (resp. negative) if $\langle A u, u\rangle \geq 0$ (resp. $\langle A u, u\rangle \leq 0)$ for all $u, v \in \operatorname{Dom}(A)$.

\subsubsection{Function Spaces}

Depending on assumptions of the problem, we can look for solutions of a PDE in different Banach spaces.

Recall that a function $u: \Omega \rightarrow \mathbb{R}$ is called Hölder continuous with order $0<\gamma \leq 1$, if

$$
|u(x)-u(y)| \leq C|x-y|^{\gamma} \quad \text { for } x, y \in \Omega
$$

for some constant $C$. Ideally, for the second-order elliptic equation (2.3), we are looking for a classical solution $u$ that, together with all its partial derivatives $D^{\alpha} u$ of second orders, $|\alpha| \leq 2$, is continuous, or even Hölder continuous. In this case we can choose the desired Banach spaces as follows.

Definition 2.2.12. Let $C^{k}(\Omega)$ be the linear space consisting of functions $u$ that, together with all their partial derivatives $D^{\alpha} u$ of orders $|\alpha| \leq k$, are continuous on $\Omega$, and let $C^{k}(\bar{\Omega})$ be the space of all functions $u \in C^{k}(\Omega)$ for which $D^{\alpha} u$ is uniformly continuous on $\Omega$, for $0 \leq|\alpha| \leq k$. Then $C^{k}(\bar{\Omega})$ is a Banach space with the norm given by

$$
\|u\|_{C^{k}(\Omega)}=\max _{0 \leq|\alpha| \leq k} \sup _{x \in \Omega}\left|D^{\alpha} u(x)\right|
$$

(See [1, p.10]).

Definition 2.2.13 (Hölder spaces). For a continuous and bounded function $u$ : $\Omega \rightarrow \mathbb{R}$, set

$$
\|u\|_{C(\bar{\Omega})}:=\sup _{x \in \Omega}|u(x)|
$$


and

$$
[u]_{C^{0, \gamma}(\bar{\Omega})}:=\sup _{\substack{x, y \in \Omega \\ x \neq y}}\left\{\frac{|u(x)-u(y)|}{|x-y|^{\gamma}}\right\} .
$$

Then, the Hölder space $C^{k, \gamma}(\bar{\Omega})$ consists of all functions $u \in C^{k}(\bar{\Omega})$ for which the norm

$$
\|u\|_{C^{k, \gamma}(\bar{\Omega})}:=\sum_{|\alpha| \leq k}\left\|D^{\alpha} u\right\|_{C(\bar{\Omega})}+\sum_{|\alpha|=k}\left[D^{\alpha} u\right]_{C^{0, \gamma}(\bar{\Omega})},
$$

is finite, which means the $k$-th partial derivative are bounded and Hölder continuous of order $\gamma$. (see[21, p.254]).

By definition, solutions belonging to Hölder spaces need to be sufficiently smooth. However, in many cases, including our problem (1.1), it is not possible to find such a classical solution for a PDE. The appropriate spaces to look for a less smooth solution are Sobolev spaces. Sobolev spaces consist of functions that satisfy a weaker version of the notion of differentiability. In other words, instead of being continuously differentiable, their weak derivative is well-defined. Hence, in order to introduce Sobolev spaces, first we need to define the notion of weak derivative.

Recall that $C_{0}^{\infty}(\Omega)$ represents the space of functions $\varphi: \Omega \rightarrow \mathbb{R}$ which are infinitely many times continuously differentiable and have compact support in $\Omega$, we define a weak derivative of a function $u$ as follows.

Definition 2.2.14 (Weak derivative). Suppose $u, v \in L_{l o c}^{1}(\Omega)$ and $\alpha$ is a multiindex. We say that $v$ is the $\alpha^{\text {th }}$-weak partial derivative of $u$, written

$$
D^{\alpha} u=v
$$

provided

$$
\int_{\Omega} u D^{\alpha} \varphi d x=(-1)^{|\alpha|} \int_{\Omega} v \varphi d x
$$

for all test functions $\varphi \in C_{0}^{\infty}(\Omega)$ (see[21, p.256]). 
Analogous to the space $C^{k}(\Omega)$, Sobolev spaces are defined in the following manner.

Definition 2.2.15 (Sobolev Spaces). The Sobolev space $W^{k, p}(\Omega)$, consists of all locally summable functions $u: \Omega \rightarrow \mathbb{R}$ such that for each multi-index $\alpha$ with $|\alpha| \leq k, D^{\alpha} u$ exists in the weak sense and belongs to $L^{p}(\Omega)$ (see [21, p.258]).

Definition 2.2.16. If $u \in W^{k, p}(\Omega)$, we define its norm to be

$$
\|u\|_{W^{k, p}(\Omega)}:= \begin{cases}\left(\sum_{|\alpha| \leq k} \int_{\Omega}\left|D^{\alpha} u\right|^{p} d x\right)^{\frac{1}{p}} & (1 \leq p<\infty), \\ \sum_{|\alpha| \leq k} \operatorname{ess}_{-\sup _{\Omega}\left|D^{\alpha} u\right|}(p=\infty),\end{cases}
$$

It is worth noting that the Sobolev spaces with the above norm are Banach Spaces (see [21] for details).

One of the subsets of Sololev spaces which are of most use when we are solving Dirichlet boundary problems (2.3), with $u=0$ on $\partial \Omega$, are $W_{0}^{k, p}(\Omega)$ spaces defined to be the closure of $C_{0}^{\infty}(\Omega)$ in $W^{k, p}(\Omega)$. In addition, as for $p=2$, Sobolev spaces are Hilbert spaces, we sometimes write $H^{k}(\Omega)=W^{k, 2}(\Omega)$ for $k=0,1, \ldots$.

\subsubsection{Solutions of a Partial Differential Equation}

Based on the spaces on which the PDE operator is defined, we can find different kinds of solutions. Hence, we give the following definitions for solutions of a PDE.

Definition 2.2.17 (Classical solution). A function $u \in C^{2}(\Omega) \cap C^{0}(\bar{\Omega})$ that satisfies (2.3) is called a classical solution for the PDE.

Definition 2.2.18 (Weak solution). Assume that $a^{i j}, b^{i}, c^{i}, d \in L^{\infty}(\Omega)$ for $i, j=$ $1, \ldots, n$ and set $f \in H^{-1}(\Omega)$, the dual space of $H_{0}^{1}(\Omega)$. The bilinear form $B[\cdot, \cdot]$ associated with the divergence form elliptic operator $L$ defined by (2.4) is

$$
B[u, v]:=\int_{\Omega} \sum_{i, j=1}^{n}\left(a^{i j}(x) u_{x_{j}}+b^{i}(x) u\right) v_{x_{i}}-\sum_{i=1}^{n} c^{i}(x) u_{x_{i}} v-d(x) u v d x
$$


for $u, v \in H_{0}^{1}(\Omega)$.

We say that $u \in H_{0}^{1}(\Omega)$ is a weak solution of the boundary-value problem (2.3) if

$$
B[u, v]=\langle f, v\rangle,
$$

for all $v \in H_{0}^{1}(\Omega)$, where $\langle\cdot, \cdot\rangle$ denotes the pairing between $H_{0}^{1}(\Omega)$ and $H^{-1}(\Omega)$ (see [21, p.315]).

Note that every classical solution of (2.3) is also a weak solutions.

In addition to classical solutions and weak solutions, we can define strong solutions for second order elliptic equations as follows.

Definition 2.2.19 (Strong solution). Consider the equation (2.3) where $L$ is in nondivergence form (2.5), with coefficient $a^{i j}, b^{i}, c \in L^{\infty}(\Omega)$. A function $u \in$ $W^{2, p}(\Omega)$, for some $p>1$, that satisfies the equation (2.3) almost everywhere in $\Omega$ is called a strong solution for the equation.

Up until now we have introduced the different kinds of solutions of linear elliptic PDEs of second order. These definitions can be extended appropriately for the semilinear elliptic equations. In this work we find either weak or strong solutions for the semilinear problem (1.1). In the remainder of this chapter we will be more specific and review some results which we need to apply along with the new variational principle in order to solve the problem (1.1).

\subsection{Inequalities in Sobolev Spaces}

\subsubsection{Standard Sobolev Embeddings}

We start with the standard compactness and embedding results in Sobolev spaces. Recall that a Banach space $V$ with norm $\|\cdot\|_{V}$ is said to be continuously embedded in the Banach space $W$ with norm $\|\cdot\|_{W}$, denoted by $V \hookrightarrow W$, if $V \subset W$ and 
there exists a constant $C \geq 0$ such that

$$
\|u\|_{W} \leq C\|u\|_{V},
$$

for all $u \in V$. Moreover, the embedding $V \hookrightarrow W$ is called compact, if it is continuous and every bounded sequence in $V$ has a strongly convergent subsequence in $W$.

In the proof of the main results of this work, the following embeddings provide us either with compactness needed for finding a critical point of a functional, or with the desired estimate of a solution of the corresponding linear equation. The following theorems are proved in [1].

Theorem 2.3.1 (The Rellich-Kondrachov theorem). Let $\Omega$ be a bounded domain in $\mathbb{R}^{n}$, with locally Lipschitz boundary. Let $k \geq 0$ and $m \geq 1$ be integers, and let $1 \leq p<\infty$

Part I If $m p \leq n$, then the following embeddings are compact:

$$
\begin{array}{ll}
W^{k+m, p}(\Omega) \hookrightarrow W^{k, q}(\Omega) & \text { if } \quad 1 \leq q<\frac{n p}{n-m p}, \\
W^{k+m, p}(\Omega) \hookrightarrow W^{k, q}(\Omega) & \text { if } \quad n=m p, \quad \text { and } \quad 1 \leq q<\infty .
\end{array}
$$

Part II If $m p>n$, then the following embeddings are compact:

$$
\begin{aligned}
W^{k+m, p}(\Omega) & \hookrightarrow C_{B}^{k}(\Omega) \\
W^{k+m, p}(\Omega) & \hookrightarrow W^{k, q}(\Omega) \quad \text { if } 1 \leq q<\infty .
\end{aligned}
$$

where $C_{B}^{k}(\Omega):=\left\{u \in C^{k}(\Omega) ; D^{\alpha} u \in L^{\infty}(\Omega)\right.$ for $\left.|\alpha| \leq k\right\}$.

Part III If $\Omega$ is an arbitrary domain in $\mathbb{R}^{n}$, the given embeddings are compact provided $W^{k+m, p}(\Omega)$ is replaced by $W_{0}^{k+m, p}(\Omega)$.

Theorem 2.3.2 (The Sobolev embeddings theorem). Let $\Omega$ be a bounded domain in $\mathbb{R}^{n}$. Let $k \geq 0$ and $m \geq 1$ be integers and $1 \leq p \leq \infty$. 
Part I Suppose that $\Omega$ has a locally Lipschitz boundary.

Case $A$ If either $m p>n$ or $m=n$ and $p=1$, then

$$
W^{k+m, p}(\Omega) \hookrightarrow C_{B}^{k}(\Omega)
$$

Moreover,

$$
W^{k+m, p}(\Omega) \hookrightarrow W^{k, q}(\Omega) \quad \text { for } 1 \leq q \leq \infty,
$$

and, in particular,

$$
W^{m, p}(\Omega) \hookrightarrow L^{q}(\Omega) \quad \text { for } 1 \leq q \leq \infty .
$$

Case B. If $m p=n$, then

$$
W^{k+m, p}(\Omega) \hookrightarrow W^{k, q}(\Omega), \quad \text { for } 1 \leq q<\infty,
$$

and, in particular,

$$
W^{m, p}(\Omega) \hookrightarrow L^{q}(\Omega), \quad \text { for } 1 \leq q<\infty .
$$

Case C. If $m p<n$, then

$$
W^{k+m, p}(\Omega) \hookrightarrow W^{k, q}(\Omega), \quad \text { for } 1 \leq q \leq \frac{n p}{n-m p} .
$$

In particular,

$$
W^{m, p}(\Omega) \hookrightarrow L^{q}(\Omega), \quad \text { for } 1 \leq q \leq \frac{n p}{n-m p} .
$$

The embedding constants for the embeddings above depend only on $n, m, p, q, k$.

Part II All the embeddings in the Part I are valid for arbitrary domains $\Omega$ if the $W$-space undergoing the embedding is replaced with the corresponding $W_{0}$-space. (See Theorem 4.12 in $[1, p .85]$ for a proof). 


\subsubsection{Sobolev Embeddings for Functions of Power Forms}

Applying the preceding standard results to our case, we can prove interesting results about the regularity of a power function $u^{p-1}$ of smooth function $u \in W^{k+2,2}(\Omega)$. These results also assist us in estimating the solution of the corresponding linear equation to our problem (1.1).

Recall that Hölder's inequality implies that for $1 \leq p, p^{\prime} \leq \infty$, with $1 / p+$ $1 / p^{\prime}=1$, if $u \in L^{p}(\Omega)$ and $v \in L^{p^{\prime}}(\Omega)$ then we have

$$
\int_{\Omega}|u v| d x \leq\|u\|_{L^{p}(\Omega)}\|v\|_{L^{p^{\prime}}(\Omega)} .
$$

We first prove the following proposition via direct application of Hölder's inequality and Sobolev embeddings.

Proposition 2.3.1. Let $p>2$ be a real number and $k$ be an integer with $0 \leq k \leq$ $p-1$. Let $u \in W^{k+2,2}(\Omega)$ where $\Omega$ is a bounded domain in $\mathbb{R}^{n}$ with $\partial \Omega \in C^{k+2}$. Furthermore, assume that $m \in\{0, \ldots, k\}$ and $s_{1}, \ldots, s_{k} \in\{0, \ldots, m\}$ satisfying $s_{1}+s_{2}+\cdots+s_{k}=m$ and $1 s_{1}+2 s_{2}+\cdots+k s_{k}=k$. If either of following assertions holds,

i) $k>1$ and either

i-a) $n \leq 2(k+2)$, or

i-b) $2(k+2)<n \leq 2(k+2)+\frac{4}{k-1}$, and $p-1 \leq \frac{n-2 k}{n-2(k+2)}$.

ii) $k=0,1$ and either

ii-a) $n \leq 2(k+2)$, or

ii-b) $n>2(k+2)$, and $p-1 \leq \frac{n-2 k}{n-2(k+2)}$,

then there exists a constant $C=C(p, \Omega, m)$ independent of $u$ such that

$$
\left\|u^{p-1-m}|D u|^{s_{1}}\left|D^{2} u\right|^{s_{2}} \cdots\left|D^{k} u\right|^{s_{k}}\right\|_{L^{2}(\Omega)} \leq C\|u\|_{W^{k+2,2}(\Omega)}^{p-1} .
$$


Proof. Let $j \leq m$ be an integer such that there exist $i_{1}, i_{2}, \ldots, i_{j} \in\{1, \ldots, k\}$ with $i_{1}<i_{2}<\cdots<i_{j}$, and that $s_{i_{1}}, s_{i_{2}}, \ldots, s_{i_{j}} \neq 0$ where $s_{i_{1}}+s_{i_{2}}+\cdots+s_{i_{j}}=m$ and $i_{1} s_{i_{1}}+i_{2} s_{i_{2}}+\cdots+i_{j} s_{i_{j}}=k$. We shall show that

$$
\left\|u^{p-1-m}\left|D^{i_{1}} u\right|^{s_{i_{1}}}\left|D^{i_{2}} u\right|^{s_{i_{2}}} \ldots\left|D^{i_{j}} u\right|^{s_{i_{j}}}\right\|_{L^{2}(\Omega)} \leq C\|u\|_{W^{k+2,2}(\Omega)}^{p-1}
$$

for an appropriate constant $C$. By applying the general Hölder's inequality to the left hand side of (2.18), we obtain

$$
\begin{gathered}
\left(\int_{\Omega}\left(u^{p-1-m}\left|D^{i_{1}} u\right|^{s_{i_{1}}}\left|D^{i_{2}} u\right|^{s_{i_{2}}} \ldots\left|D^{i_{j}} u\right|^{s_{i_{j}}}\right)^{2} d x\right)^{\frac{1}{2}} \leq \\
\left(\int_{\Omega}|u|^{2(p-1-m) r_{0}} d x\right)^{\frac{1}{2 r_{0}}}\left(\int_{\Omega}\left|D^{i_{1}} u\right|^{2 s_{i_{1}} r_{1}} d x\right)^{\frac{1}{2 r_{1}}} \cdots\left(\int_{\Omega}\left|D^{i_{j}} u\right|^{2 s_{i_{j}} r_{j}} d x\right)^{\frac{1}{2 r_{j}}},
\end{gathered}
$$

where $\frac{1}{r_{0}}+\frac{1}{r_{1}}+\cdots+\frac{1}{r_{j}}=1$ and $1 \leq r_{0}, \ldots, r_{j} \leq \infty$. Recalling that $u \in W^{k+2,2}(\Omega)$, we wish to choose $r_{0}, \ldots, r_{j}$ in a way that every term in (2.19) is controlled by $\|u\|_{W^{k+2,2}(\Omega)}$. As $\partial \Omega \in C^{k+2}$, we can make use of the embeddings in Theorems 2.3.1 and 2.3.2 to find various spaces where $W^{k+2,2}(\Omega)$ is embedded into. Considering the fact that these embeddings depend directly on $n$, we verify the result in three different ranges of $n$; for $n=2(k+2), n<2(k+2)$ and $2(k+2)<n \leq 2(k+2)+\frac{4}{k-1}$.

i) $k>1$ :

$\boldsymbol{i}$-a.1) Let $n=2(k+2)$.

In case of $r_{0}$, we consider the embedding $W^{k+2,2}(\Omega) \hookrightarrow L^{q}(\Omega)$ for $1 \leq q<\infty$. Applying this in (2.19), we can see that $u \in L^{q}(\Omega)$ for any choice of $1 \leq q<\infty$, so for any value of $r_{0} \neq 2(p-1-m)$ we have

$$
\left(\int_{\Omega}|u|^{2(p-1-m) r_{0}} d x\right)^{\frac{1}{2 r_{0}}}=\|u\|_{L^{2(p-1-m) r_{0}(\Omega)}}^{p-1-m} \leq C_{0}\|u\|_{W^{k+2,2}(\Omega)}^{p-1-m} .
$$

Furthermore, as $n=2(k+2)$ implies $2\left(k+2-i_{l}\right) \leq n$ for $1 \leq l \leq j$, we can apply part I-C of Theorem 2.3.2 and choose $r_{l}$ in such a way that the embedding $W^{k+2,2}(\Omega)=W^{i_{l}+\left(k+2-i_{l}\right), 2}(\Omega) \hookrightarrow W^{i_{l}, 2 s_{i} r_{l}}(\Omega)$ holds true. 
By setting $2 s_{i_{l}} r_{l}=2 n /\left(n-2\left(k+2-i_{l}\right)\right)$, the largest value for which the continuous embedding can be applied, we find $r_{l}$ for $l=1, \ldots, j$ such that

$$
r_{l}=\frac{n}{\left(n-2\left(k+2-i_{l}\right)\right) s_{i_{l}}} .
$$

In order to use Hölder's inequality, we should verify that each $r_{l}>1$ and $0<$ $\sum_{r=1}^{j} \frac{1}{r_{l}}<1$. Note that

$$
\begin{aligned}
\sum_{l=1}^{j} \frac{1}{r_{l}} & =\sum_{l=1}^{j} \frac{\left(n-2\left(k+2-i_{l}\right)\right) s_{i_{l}}}{n} \\
& =\frac{(n-2(k+2)) \sum_{l=1}^{j} s_{i_{l}}+2 \sum_{l=1}^{j} i_{l} s_{i_{l}}}{n} \\
& =\frac{(n-2(k+2)) m+2 k}{n}=\frac{2 k}{2(k+2)},
\end{aligned}
$$

which is obviously positive and less than one. Also, note that each $r_{l}$ is positive as $n=2(k+2)$, which means that $n>2\left(k+2-i_{l}\right)$ for all $l$. Therefore, as $r_{l}>0$ for all $l$ and $\sum_{l=1}^{j} \frac{1}{r_{l}}<1$, we can conclude that $r_{l}>1$ for all $l$.

Hence, we do not have any issues in using Hölder's inequality. It also follows that for $l=1, \ldots, j$,

$$
\left(\int_{\Omega}\left|D^{i_{l}} u\right|^{2 s_{i_{l}} r_{l}} d x\right)^{\frac{1}{2 r_{l}}} \leq\|u\|_{W^{i_{l}, 2 s_{i} r_{l}(\Omega)}}^{s_{i_{l}}} \leq C_{l}\|u\|_{W^{k+2,2}(\Omega)}^{s_{i_{l}}} .
$$

Now, we choose $r_{0}$ such that $\frac{1}{r_{0}}=1-\sum_{l=1}^{j} \frac{1}{r_{l}}$. Since $\sum_{l=1}^{j} \frac{1}{r_{l}}<1$, one has that $r_{0}>1$ as well. Hence, combining (2.20), (2.21), and (2.19) we obtain that

$$
\begin{aligned}
& \left(\int_{\Omega}\left(u^{p-1-m}\left|D^{i_{1}} u\right|^{s_{i_{1}}}\left|D^{i_{2}} u\right|^{s_{i_{2}}} \ldots\left|D^{i_{j}} u\right|^{s_{i_{j}}}\right)^{2} d x\right)^{\frac{1}{2}} \\
& \leq\|u\|_{L^{2(p-1-m) r_{0}(\Omega)}}^{p-1-m}\|u\|_{W^{i_{1}, 2 s_{i_{1}} r_{1}(\Omega)}}^{s_{i_{1}}} \cdots\|u\|_{W^{i_{j}, 2 s_{i_{j}} r_{j}}(\Omega)}^{s_{i_{j}}} \\
& \leq C_{0}\|u\|_{W^{k+2,2}(\Omega)}^{p-1-m} C_{1}\|u\|_{W^{k+2,2}(\Omega)}^{s_{1_{1}}} \cdots C_{j}\|u\|_{W^{k+2,2}(\Omega)}^{s_{i_{j}}} \\
& \leq C\|u\|_{W^{k+2,2}(\Omega)}^{p-1} \text {, }
\end{aligned}
$$

where $C=C_{0} C_{1} \ldots C_{j}$. Therefore, we have the result for this case.

$\boldsymbol{i}$-a.2) Let $n<2(k+2)$. 
For this range of $n$, we have the embedding $W^{k+2,2}(\Omega) \hookrightarrow C_{B}^{0}(\Omega)$, which follows from Theorem 2.3.2. As $\Omega$ is bounded, $u \in W^{k+2,2}(\Omega)$ implies that $u \in$ $C_{B}^{0}(\Omega)$, for any choice of $r_{0}$ the first integral in the right hand side of $(2.19)$ is controlled by $\|u\|_{W^{k+2,2}(\Omega)}$.

To deal with the rest of the terms in (2.19), we need to investigate it for different ranges of $n$ in order to use the desired embeddings. For $2\left(k+2-i_{1}\right)<n<$ $2(k+2)$ where $i_{1}$ is the lowest order of derivative in $(2.19)$, since $2\left(k+2-i_{1}\right)<n$ we can use the embedding $W^{k+2,2}(\Omega) \hookrightarrow W^{i_{l}, 2 s_{i} r_{l}}(\Omega)$ for $l=1, \ldots, j$. Hence, as in the previous case, we choose $r_{l}$ for $l=1, \ldots, j$ such that

$$
r_{l}=\frac{n}{\left(n-2\left(k+2-i_{l}\right)\right) s_{i_{l}}} .
$$

As shown before, for $l=1, \ldots, j$ we have $r_{l}>1$. In particular, we can also show that $0<\sum_{r=1}^{j} \frac{1}{r_{l}}<1$. Indeed,

$$
\begin{aligned}
\sum_{l=1}^{j} \frac{1}{r_{l}} & =\sum_{l=1}^{j} \frac{\left(n-2\left(k+2-i_{l}\right)\right) s_{i_{l}}}{n} \\
& =\frac{(n-2(k+2)) \sum_{l=1}^{j} s_{i_{l}}+2 \sum_{l=1}^{j} i_{l} s_{i_{l}}}{n} \\
& =\frac{(n-2(k+2)) m+2 k}{n} .
\end{aligned}
$$

But (2.22) is positive as from the assumption we have

$$
n-2\left(k+2-i_{1}\right)>0 \text {. }
$$

So, we can deduce that

$$
\begin{aligned}
m(n-2(k+2))+2 k=m\left(n-2\left(k+2-\frac{k}{m}\right)\right) & =m\left(n-2\left(k+2-\frac{\sum_{l=1}^{j} i_{l} s_{i_{l}}}{\sum_{l=1}^{j} s_{i_{l}}}\right)\right) \\
& \geq m\left(n-2\left(k+2-\frac{i_{1} \sum_{l=1}^{j} s_{i_{l}}}{\sum_{l=1}^{j} s_{i_{l}}}\right)\right) \\
& =m\left(n-2\left(k+2-i_{1}\right)\right) \\
& >0 .
\end{aligned}
$$


Also, for $(2.22)$ to be less than one we must have $((n-2(k+2)) m+2 k) / n<1$ or equivalently

$$
n<2(k+2)+\frac{4}{m-1}
$$

which is true as we already have $n<2(k+2)$. Now, with these choices of $r_{l}$, we can apply the embedding $W^{k+2,2}(\Omega) \hookrightarrow W^{i_{l}, 2 s_{i} r_{l}}(\Omega)$ and obtain that for $l=1, \ldots, j$,

$$
\left(\int_{\Omega}\left|D^{i_{l}} u\right|^{2 s_{i_{l}} r_{l}} d x\right)^{\frac{1}{2 r_{l}}} \leq\|u\|_{W^{i_{l}, 2 s_{i} r_{l}(\Omega)}}^{s_{i_{l}}} \leq C_{l}\|u\|_{W^{k+2,2}(\Omega)}^{s_{i_{l}}} .
$$

Next, we choose $r_{0}$ such that $\frac{1}{r_{0}}=1-\sum_{l=1}^{j} \frac{1}{r_{l}}$. Using the embedding $W^{k+2,2}(\Omega) \hookrightarrow$ $C_{B}^{0}(\Omega)$ for $r_{0}$ we have

$$
\left(\int_{\Omega} u^{2(p-1-m) r_{0}} d x\right)^{\frac{1}{2 r_{0}}} \leq|\Omega|^{\frac{1}{2 r_{0}}}\|u\|_{C_{B}^{0}(\Omega)}^{p-1-m} \leq C_{0}|\Omega|^{\frac{1}{2 r_{0}}}\|u\|_{W^{k+2,2}(\Omega)}^{p-1-m} \leq C_{0}^{\prime}\|u\|_{W^{k+2,2}(\Omega)}^{p-1-m},
$$

where $|\Omega|$ is the measure of the set $\Omega$. Hence, putting together $(2.24),(2.25)$ and (2.19) we obtain

$$
\begin{aligned}
& \left(\int_{\Omega}\left(u^{p-1-m}\left|D^{i_{1}} u\right|^{s_{i_{1}}}\left|D^{i_{2}} u\right|^{s_{2}} \cdots\left|D^{i_{j}} u\right|^{s_{i_{j}}}\right)^{2} d x\right)^{\frac{1}{2}} \\
& \leq|\Omega|^{\frac{1}{2 r_{0}}}\|u\|_{C_{B}^{0}(\Omega)}^{p-1-m}\|u\|_{W^{i_{1}, 2 s_{i_{1}} r_{1}}(\Omega)}^{s_{i_{1}}} \cdots\|u\|_{W^{i_{j}, 2 s_{i_{j}} r_{j}}(\Omega)}^{s_{i_{j}}} \cdots \\
& \leq C_{0}^{\prime}\|u\|_{W^{k+2,2}(\Omega)}^{p-1-m} C_{1}\|u\|_{W^{k+2,2}(\Omega)}^{s_{i_{1}}} \cdots C_{j}\|u\|_{W^{k+2,2}(\Omega)}^{s_{i_{j}}} \\
& \leq C\|u\|_{W^{k+2,2}(\Omega)}^{p-1} \text {, }
\end{aligned}
$$

where $C=C_{0}^{\prime} C_{1} \ldots C_{j}$.

Now, we deal with $(2.19)$ for $2\left(k+2-i_{2}\right)<n \leq 2\left(k+2-i_{1}\right)$. Note that for this range, we have $n \leq 2\left(k+2-i_{1}\right)$. So in case of $i_{1}$, by virtue of part I-A of Theorem 2.3.2, since $2\left(k+2-i_{2}\right)<n<2\left(k+2-i_{1}\right)$ we can use either the embedding $W^{k+2,2}(\Omega)=W^{i_{1}+\left(k+2-i_{1}\right), 2}(\Omega) \hookrightarrow C_{B}^{i_{1}}(\Omega)$ or $W^{k+2,2}(\Omega)=W^{i_{1}+\left(k+2-i_{1}\right), 2}(\Omega) \hookrightarrow$ $W^{i_{1}, q}(\Omega)$ for any $1 \leq q<\infty$. Furthermore, if $n=2\left(k+2-i_{1}\right)$ from part IB of Theorem 2.3.2, we have the embedding $W^{k+2,2}(\Omega)=W^{i_{1}+\left(k+2-i_{1}\right), 2}(\Omega) \hookrightarrow$ $W^{i_{1}, q}(\Omega)$ for any $1 \leq q<\infty$. Therefore, to be consistent we use $W^{k+2,2}(\Omega) \hookrightarrow$ 
$W^{i_{1}, q}(\Omega)$ for $i_{1}$. Using a similar argument as in the previous cases, since we have $u \in C_{B}^{0}(\Omega)$ and $D^{i_{1}} u \in W^{i_{1}, q}(\Omega)$ for $1 \leq q<\infty$, for any choices of $r_{0}$ and $r_{1}$ the first two terms in (2.19) are controlled by $\|u\|_{W^{k+2,2(\Omega)}}$. Also, as $2\left(k+2-i_{2}\right)<n$, we can use the embeddings $W^{k+2,2}(\Omega) \hookrightarrow W^{i_{l}, 2 s_{i} r_{l}}(\Omega)$ for $l=2, \ldots, j$. Hence, we can choose $r_{l}$ for $j=2, \ldots l$ as before,

$$
r_{l}=\frac{n}{\left(n-2\left(k+2-i_{l}\right)\right) s_{i_{l}}} .
$$

Obviously, for each $r_{l}$ we have $r_{l}>1$. To verify that $0<\sum_{r=2}^{j} \frac{1}{r_{l}}<1$ we need to have

$$
\sum_{l=2}^{j} \frac{1}{r_{l}}=\frac{(n-2(k+2)) m+2 k-\left(n-2\left(k+2-i_{1}\right)\right) s_{i_{1}}}{n}<1 .
$$

It is easily seen that (2.26) holds if and only if

$$
n<2(k+2)+\frac{4+2 i_{1} s_{i_{1}}}{m-1-s_{i_{1}}},
$$

which is already satisfied as in this case we have $m-1>s_{i_{1}}$ and $n \leq 2\left(k+2-i_{1}\right)$. Furthermore, we choose $r_{0}$ and $r_{1}$ such that $\frac{1}{r_{0}}+\frac{1}{r_{1}}=1-\sum_{r=1}^{j} \frac{1}{r_{l}}$. Then by using the mentioned embeddings we obtain

$$
\begin{aligned}
& \left(\int_{\Omega}\left(u^{p-1-m}\left|D^{i_{1}} u\right|^{s_{i_{1}}}\left|D^{i_{2}} u\right|^{s_{i_{2}}} \cdots\left|D^{i_{j}} u\right|^{s_{i_{j}}}\right)^{2} d x\right)^{\frac{1}{2}} \\
& \quad \leq|\Omega|^{\frac{1}{2 r_{0}}}\|u\|_{C_{B}^{0}(\Omega)}^{p-1-m}\|u\|_{W^{i_{1}, 2 s_{i_{1}} r_{1}}(\Omega)}^{s_{i_{1}}}\|u\|_{W^{i_{2}, 2 s_{i_{2} r_{2}}}(\Omega)}^{s_{i_{2}}} \cdots\|u\|_{W^{i_{j}, 2 s_{i_{j}} r_{j}}(\Omega)}^{s_{i_{j}}} \\
& \quad \leq C_{0}^{\prime}\|u\|_{W^{k+2,2}(\Omega)}^{p-1-m} C_{1}\|u\|_{W^{k+2,2}(\Omega)}^{s_{i_{1}}} C_{2}\|u\|_{W^{k+2,2}(\Omega)}^{s_{i_{2}}} \cdots C_{j}\|u\|_{W^{k+2,2}(\Omega)}^{s_{i_{j}}} \\
& \quad \leq C\|u\|_{W^{k+2,2(\Omega)}}^{p-1},
\end{aligned}
$$

for an appropriate constant $C$.

Similarly for $l=2, \ldots, j-1$ and $2\left(k+2-i_{l+1}\right)<n \leq 2\left(k+2-i_{l}\right)$, we see that either $u \in C_{B}^{i_{l}}(\Omega)$, which means $D^{i_{l}} u \in C_{B}^{0}(\Omega)$, or $u \in W^{i_{l}, q}(\Omega)$ for $1 \leq q<\infty$. Finally, for $n<4=2(k+2-k)$ we have $u \in C_{B}^{k}(\Omega)$. Then, we obtain

$$
\left(\int_{\Omega}\left(u^{p-1-m}\left|D^{i_{1}} u\right|^{s_{i_{1}}}\left|D^{i_{2}} u\right|^{s_{i_{2}}} \ldots\left|D^{i_{j}} u\right|^{s_{i_{j}}}\right)^{2} d x\right)^{\frac{1}{2}}
$$




$$
\begin{aligned}
& \leq|\Omega|^{\frac{1}{2 r_{0}}}\|u\|_{C_{B}^{0}(\Omega)}^{p-1-m}|\Omega|^{\frac{1}{2 r_{1}}}\left\|D^{i_{1}} u\right\|_{C_{B}^{0}(\Omega)}^{s_{i_{1}}} \cdots|\Omega|^{\frac{1}{2 r_{j}}}\left\|D^{i_{j}} u\right\|_{C_{B}^{0}(\Omega)}^{s_{i_{j}}} \\
& \leq C_{0}^{\prime}\|u\|_{W^{k+2,2}(\Omega)}^{p-1-m} C_{1}^{\prime}\|u\|_{W^{k+2,2}(\Omega)}^{s_{i_{1}}} C^{\prime}{ }_{2}\|u\|_{W^{k+2,2}(\Omega)}^{s_{i_{2}}} \cdots C^{\prime}{ }_{j}\|u\|_{W^{k+2,2}(\Omega)}^{s_{i_{j}}} \\
& \leq C\|u\|_{W^{k+2,2}(\Omega)}^{p-1}
\end{aligned}
$$

as desired.

$\boldsymbol{i}$-b) Let $2(k+2)<n \leq 2(k+2)+\frac{4}{k-1}$ and $p-1 \leq(n-2 k) /(n-2(k+2))$.

In case of $r_{0}$, as we have $2(k+2)<n$ and $\partial \Omega \in C^{k+2}$, we can apply the embedding $W^{k+2,2}(\Omega) \hookrightarrow L^{\frac{2 n}{n-2(K+2)}}(\Omega)$, which we have from Theorem 2.3.2, Part I-C. As a result, we need to set $2(p-1-m) r_{0}=2 n / n-2(k+2)$, and consequently we obtain

$$
r_{0}=\frac{n}{(n-2(k+2))(p-1-m)} .
$$

Before we use $r_{0}$, we need to verify that it is greater than one, so if we set $r_{0}>1$, we find the condition $p-1<((m+1) n-2 m(k+2)) /(n-2(k+2))$, which is already satisfied as by assumption we have $1<p-1 \leq(n-2 k) /(n-2(k+2))$, and $2(k+2)<n$. Thus, for the first term in (2.19), we get

$$
\begin{aligned}
\left(\int_{\Omega} u^{2(p-1-m) r_{0}} d x\right)^{\frac{1}{2 r_{0}}} & =\left(\int_{\Omega} u^{\frac{2 n}{n-2(k+2)}} d x\right)^{\frac{(n-2(k+2))(p-1-m)}{2 n}} \\
& =\|u\|_{L^{\frac{2 n}{n-2(k+2)}(\Omega)}}^{p-1-m} \leq C_{0}\|u\|_{W^{k+2,2}(\Omega)}^{p-1-m}
\end{aligned} .
$$

Moreover, similar to the previous part as $2(k+2)<n$ implies $2\left(k+2-i_{l}\right) \leq n$, for $l=1, \ldots j-1$, we use the embedding $W^{k+2,2}(\Omega) \hookrightarrow W^{i_{l}, 2 s_{i} r_{l}}(\Omega)$, and set $r_{l}$ as follows

$$
r_{l}=\frac{n}{\left(n-2\left(k+2-i_{l}\right)\right) s_{i_{l}}} .
$$

By setting $r_{l}>1$ for $l=1, \ldots j-1$, we find the condition $n<2(k+2)+$ $\left(2\left(k+2-i_{l}\right) s_{i_{l}}\right) /\left(s_{i_{l}}-1\right)$, which is satisfied for our case since we already have $n \leq 2(k+2)+4 /(k-1)$. Also, to see if $\sum_{l=1}^{j} \frac{1}{r_{l}}<1$ we set

$$
\sum_{l=1}^{j-1} \frac{1}{r_{l}}=\frac{(n-2(k+2))\left(m-s_{i_{j}}\right)+2\left(k-i_{j} s_{i_{j}}\right)+(n-2(k+2)(p-1-m)}{n}<1
$$


So, we obtain

$$
p-1<\frac{\left(n-2 k+2 i_{j} s_{i_{j}}\right)}{(n-2(k+2))}+s_{i j} .
$$

Therefore, as by assumption we have $1<p-1 \leq n-2 k /(n-2(k+2))$, we can conclude that $\sum_{l=1}^{j-1} \frac{1}{r_{l}}<1$. Now we need to find $r_{j}$ such that $\frac{1}{r_{0}}+\frac{1}{r_{1}}+\ldots+\frac{1}{r_{j}}=1$. So,

$$
r_{j}=\frac{\prod_{l=0}^{j-1} r_{l}}{\prod_{l=0}^{j-1} r_{l}-\sum_{z=0}^{j-1}\left(\prod_{\substack{l=0, l \neq z}}^{j-1} r_{l}\right)}
$$

which gives us

$$
\begin{aligned}
r_{j} & =\frac{\frac{n}{(n-2(k+2))(p-1-m)} \cdot \prod_{l=1}^{j-1} \frac{n}{\left(n-2\left(k+2-i_{l}\right)\right) s_{i_{l}}}}{\left(\frac{n}{(n-2(k+2))(p-1-m)}-1\right) \cdot \prod_{l=1}^{j-1} \frac{n}{\left(n-2\left(k+2-i_{l}\right)\right) s_{i_{l}}}-\sum_{z=1}^{j-1}\left(\frac{n}{(n-2(k+2))(p-1-m)} \prod_{\substack{l=1,1 \\
l \neq z}}^{j-1} \frac{n}{\left(n-2\left(k+2-i_{l}\right)\right) s_{i_{l}}}\right)} \\
& =\frac{n^{j}}{n^{j}-n^{j-1}(n-2(k+2))(p-1-m)-\sum_{l=1}^{j-1} n^{j-1}\left(n-2\left(k+2-i_{l}\right)\right) s_{i_{l}}} \\
& =\frac{n-(n-2(k+2))(p-1-m)-\sum_{l=1}^{j-1}(n-2(k+2)) s_{i_{l}}-2 \sum_{l=1}^{j-1} i_{l} s_{i_{l}}}{n-} .
\end{aligned}
$$

By assumption we have $\sum_{l=1}^{j} i_{l} s_{i_{l}}=k$, or equivalently $\sum_{l=1}^{j-1} i_{l} s_{i_{l}}=k-i_{j} s_{i_{j}}$, and we obtain

$$
r_{j}=\frac{n}{n-(n-2(k+2))(p-1-m)-(n-2(k+2)) \sum_{l=1}^{j-1} s_{i_{l}}-2\left(k-i_{j} s_{i_{j}}\right)} .
$$

Again, by assumption we have $\sum_{l=1}^{j} s_{i_{l}}=m$. Hence $\sum_{l=1}^{j-1} s_{i_{l}}=m-s_{i_{j}}$, and we thus get

$$
\begin{aligned}
r_{j} & =\frac{n}{n-(n-2(k+2))(p-1-m)-(n-2(k+2))\left(m-s_{i_{j}}\right)-2\left(k-i_{j} s_{i_{j}}\right)} \\
& =\frac{n-(n-2(k+2))\left(p-1-s_{i_{j}}\right)-2\left(k-i_{j} s_{i_{j}}\right)}{n-(28)}
\end{aligned}
$$


Now to control the last term in (2.19), which is $\left(\int_{\Omega}\left|D^{i_{j}} u\right|^{2 s_{i_{j}} r_{j}} d x\right)^{\frac{1}{2 r_{j}}}$, we wish to use the embedding

$$
W^{k+2,2}(\Omega) \hookrightarrow W^{i_{j}, 2 s_{i_{j}} r_{j}}(\Omega), \quad \quad \text { for } \quad 1 \leq 2 s_{i_{j}} r_{j} \leq \frac{2 n}{n-2\left(k+2-i_{j}\right)},
$$

so, we must find $p$ such that

$$
r_{j} \leq \frac{n}{\left(n-2\left(k+2-i_{j}\right)\right) s_{i_{j}}} .
$$

Therefore, substituting the value for $r_{j}$ from (2.28) yields

$$
\frac{n}{n-(n-2(k+2))\left(p-1-s_{i_{j}}\right)-2\left(k-i_{j} s_{i_{j}}\right)} \leq \frac{n}{\left(n-2\left(k+2-i_{j}\right)\right) s_{i_{j}}},
$$

and hence

$$
\begin{aligned}
& (n-2(k+2)) s_{i_{j}}+2 i_{j} s_{i_{j}} \leq n-(n-2(k+2))\left(p-1-s_{i_{j}}\right)-2\left(k-i_{j} s_{i_{j}}\right) \\
& \left(p-1-s_{i j}\right) \leq \frac{n-2 k-(n-2(k+2)) s_{i_{j}}}{n-2(k+2)} \\
& p-1 \leq \frac{n-2 k}{(n-2(k+2))} .
\end{aligned}
$$

As a result, for $p-1 \leq(n-2 k) /(n-2(k+2))$, all the terms in (2.19) are dominated by $\|u\|_{W^{k+2,2}(\Omega)}$, as for $l=1, \ldots, j$ we have

$$
\left(\int_{\Omega}\left|D^{i_{l}} u\right|^{2 s_{i_{l}} r_{l}} d x\right)^{\frac{1}{2 r_{l}}} \leq\|u\|_{W^{i_{l}, 2 s_{i} r_{l}(\Omega)}}^{s_{i_{l}}} \leq C_{l}\|u\|_{W^{k+2,2}(\Omega)}^{s_{i_{l}}}
$$

So, by using (2.27) and (2.29) in (2.19) we obtain

$$
\begin{aligned}
&\left(\int_{\Omega}\left(u^{p-1-m}\left|D^{i_{1}} u\right|^{s_{i_{1}}}\left|D^{i_{2}} u\right|^{s_{i_{2}}} \ldots\left|D^{i_{j}} u\right|^{s_{i_{j}}}\right)^{2} d x\right)^{\frac{1}{2}} \\
& \quad \leq\|u\|_{L^{\frac{2 n}{n-2(k+2)}(\Omega)}}^{p-1-m}\|u\|_{W^{i_{1}, 2 s_{i_{1}} r_{1}}(\Omega)}^{s_{i_{1}}} \ldots\|u\|_{W^{i_{j}, 2 s_{i_{j}} r_{j}}(\Omega)}^{s_{i_{j}}} \\
& \quad \leq C_{0}\|u\|_{W^{k+2,2}(\Omega)}^{p-1-m} C_{1}\|u\|_{W^{k+2,2}(\Omega)}^{s_{i_{1}}} \ldots C_{j}\|u\|_{W^{k+2,2}(\Omega)}^{s_{i_{j}}{ }^{k+2}} \\
& \leq C\|u\|_{W^{k+2,2}(\Omega)}^{p-1},
\end{aligned}
$$

which proves the claim.

By a rather similar argument one can repeat the proof for other cases, $\boldsymbol{i i}$ - $\boldsymbol{a}$ and $i i-b$. 
As a consequence of Proposition 2.3.1 we can prove the following Lemma.

Lemma 2.3.3. Let $p>2$ be a real number. Assume that $u \in W^{k+2,2}(\Omega)$ with $\Omega$ being a bounded domain in $\mathbb{R}^{n}, \partial \Omega \in C^{k+2}$ and $0 \leq k \leq p-1$. Then we have the following inequality

$$
\left\|u^{p-1}\right\|_{W^{k, 2}(\Omega)} \leq C_{p}\|u\|_{W^{k+2,2}(\Omega)}^{p-1},
$$

provided that $1<p-1 \leq(n-2 k) /(n-2(k+2))$ if $2(k+2)<n \leq 2(k+2)+$ $4 /(k-1)$, or $p>2$ if $n \leq 2(k+2)$.

Proof. To prove the result we estimate $D^{\alpha}\left(u^{p-1}\right)$, where $\alpha$ is a multi-index with $|\alpha| \leq k$. By an easy calculation, we find that for $|\alpha|=k^{\prime}$, where $0 \leq k^{\prime} \leq k$, $D^{\alpha}\left(u^{p-1}\right)$ can be written as a finite sum of terms in the following form

$$
u^{p-1-m^{\prime}}|D u|^{s_{1}}\left|D^{2} u\right|^{s_{2}} \cdots\left|D^{k^{\prime}} u\right|^{s_{k^{\prime}}},
$$

where $1 \leq m^{\prime} \leq k^{\prime}$ and $s_{1}, s_{2}, \ldots, s_{k^{\prime}} \in\left\{0,1, \ldots, m^{\prime}\right\}$ with $s_{1}+s_{2}+\cdots+s_{k^{\prime}}=m^{\prime}$ and $1 s_{1}+2 s_{2}+\cdots+k^{\prime} s_{k^{\prime}}=k^{\prime}$. Thus, we just need to prove that each term of the form (2.30) is being controlled by $\|u\|_{W^{k+2,2}(\Omega)}$.

When $k^{\prime}=k$, we have shown in Proposition 2.3.1 that for $u \in W^{k+2,2}(\Omega)$, all terms in the form (2.30) are controlled by $\|u\|_{W^{k+2,2}(\Omega)}$ as long as $2<p-1 \leq$ $(n-2 k) /(n-2(k+2))$ if $2(k+2)<n \leq 2(k+2)+4 /(k-1)$, or $p>2$ if $n \leq 2(k+2)$.

Following the same steps as in Proposition 2.3.1, similarly for $1 \leq k^{\prime}<k$, it can be proved that for $|\alpha|=k^{\prime}, D^{\alpha}\left(u^{p-1}\right)$ is dominated by $\|u\|_{W^{k+2,2}(\Omega)}$ as long as $2<p-1 \leq\left(n-2 k^{\prime}\right) /(n-2(k+2))$ if $2(k+2)<n \leq 2(k+2)+4 /(k-1)$, or $p>2$ if $n \leq 2(k+2)$.

As one can see for $n \leq 2(k+2)$, we have the result. However, if $2(k+2)<$ $n \leq 2(k+2)+4 /(k-1)$, we need to choose $p$ such that for all $0 \leq k^{\prime} \leq k$, the conditions $1<p-1 \leq\left(n-2 k^{\prime}\right) /(n-2(k+2))$ are satisfied. But it is obvious that for $0 \leq k^{\prime} \leq k$, we have

$$
\frac{(n-2 k)}{(n-2(k+2))} \leq \frac{\left(n-2 k^{\prime}\right)}{(n-2(k+2))},
$$


Hence, by choosing $1<p-1 \leq(n-2 k) /(n-2(k+2))$ we have the desired estimate.

Remark 2.3.4. Note that the assumptions on $p-1$ in Proposition 2.3.1 and Lemma 2.3.3 are equivalent to the following assumptions on $p$,

- $2<p \leq \frac{2(n-2(k+1))}{n-2(k+2)}$ if $2(k+2)<n \leq 2(k+2)+\frac{4}{k-1}$, or

- $2<p$ if $n \leq 2(k+2)$.

Following the same argument as in the proof of Proposition 2.3.1, we can show an analogous result for $u \in W^{2, d}(\Omega)$, where $d \geq 2$ is a real number.

Lemma 2.3.5. Let $p>2$ and $d \geq 2$ be real numbers. Assume that $u \in W^{2, d}(\Omega)$ with $\Omega$ being a bounded domain in $\mathbb{R}^{n}, \partial \Omega \in C^{1,1}$. Then we have

$$
\left\|u^{p-1}\right\|_{L^{d}(\Omega)} \leq C_{p}\|u\|_{W^{2, d}(\Omega)}^{p-1}
$$

provided that $1<p-1 \leq n /(n-2 d)$ if $2 d<n$, or $p>2$ if $n \leq 2 d$.

Proof. We consider the following cases,

i) For $n \leq 2 d$ : recalling parts I-A and I-B of Theorem 2.3.2, we can use the embedding $W^{2, d}(\Omega) \hookrightarrow L^{q}(\Omega)$ for $1 \leq q<\infty$. Therefore, we can set $q=d(p-1)$ to obtain

$$
\left\|u^{p-1}\right\|_{L^{d}(\Omega)}=\left(\int_{\Omega}|u|^{d(p-1)} d x\right)^{\frac{1}{d}}=\|u\|_{L^{d(p-1)}(\Omega)}^{p-1} \leq C_{p}\|u\|_{W^{2, d}(\Omega)}^{p-1},
$$

for an appropriate constant $C_{p}$.

ii) For $n>2 d$ : using the embedding $W^{2, d}(\Omega) \hookrightarrow L^{q}(\Omega)$ for $1 \leq q \leq d n /(n-2 d)$, which we have from Part I-C of Theorem 2.3.2, we find that $u \in L^{d(p-1)}(\Omega)$ for $d(p-1) \leq d n /(n-2 d)$. Hence, for $p-1 \leq n / n-2 d$ we obtain

$$
\left\|u^{p-1}\right\|_{L^{d}(\Omega)}=\left(\int_{\Omega}|u|^{d(p-1)} d x\right)^{\frac{1}{d}}=\|u\|_{L^{d(p-1)}(\Omega)}^{p-1} \leq C_{p}\|u\|_{W^{2, d}(\Omega)}^{p-1},
$$

as claimed. 
Remark 2.3.6. The assumptions on $p-1$ in Lemma 2.3.5 are equivalent to the following assumptions on $p$,

- $2<p \leq \frac{(2 n-2 d)}{n-2 d}$ if $2 d<n$, or

- $2<p$ if $n \leq 2 d$.

\subsection{Elliptic Regularity Theory}

Elliptic regularity and $L^{p}$ estimates determine the existence of higher-order weak derivatives for a weak solution of (2.3). In our approach, in order to show that the solution of a corresponding linear equation belongs to the specific convex set $K$, based on the definition of $K$ in our problems, we need to verify that this weak solution is regular enough. Hence we recall the following results from [22].

Theorem 2.4.1 (Elliptic regularity). Let $u \in W^{1,2}(\Omega)$ be a weak solution of the equation $L u=f$ in $\Omega$, where the operator $L$, defined in (2.4), is strictly elliptic in $\Omega$, the coefficients $a^{i j}, b^{i} \in C^{k, 1}(\bar{\Omega})$, the coefficients $c^{i}, d \in C^{k-1,1}(\bar{\Omega})$, and the function $f \in W^{k, 2}(\Omega), k \geq 1$. In addition, let us assume that $\partial \Omega \in C^{k+2}$ and that there exists a function $\varphi \in W^{k+2,2}(\Omega)$ for which $u-\varphi \in W_{0}^{1,2}(\Omega)$. Then we have also $u \in W^{k+2,2}(\Omega)$ and

$$
\|u\|_{W^{k+2,2}(\Omega)} \leq C\left(\|u\|_{L^{2}(\Omega)}+\|f\|_{W^{k, 2}(\Omega)}+\|\varphi\|_{W^{k+2,2}(\Omega)}\right),
$$

where $C=C\left(n, \lambda, K^{\prime}, k, \partial \Omega\right)$, and $K^{\prime}=\max \left\{\left\|a^{i j}, b^{i}\right\|_{C^{k, 1}(\bar{\Omega})},\left\|c^{i}, d\right\|_{C^{k-1,1}(\bar{\Omega})}\right\}$. If the functions $a^{i j}, b^{i}, c^{i}, d, f$ and $\varphi$ belong to $C^{\infty}(\bar{\Omega})$ and $\partial \Omega$ is of class of $C^{\infty}$, then the solution $u$ is also in $C^{\infty}(\bar{\Omega})$ (see Theorem 8.13 [22, p. 187]).

Theorem 2.4.2 ( $L^{p}$ estimates). Let $\Omega$ be a domain in $\mathbb{R}^{n}$ with a $C^{1,1}$ boundary portion $T \subset \partial \Omega$. Let $u \in W^{2, p}(\Omega), 1<p<\infty$, be a strong solution of $L u=f$ in $\Omega$ with $u=0$ on $T$, in the sense of $W^{1, p}(\Omega)$, where $f \in L^{p}(\Omega)$ in $\Omega$ and the coefficients of $L$ satisfy, for positive constants $\lambda, \Lambda$, 
- $a^{i j} \in C^{0}(\Omega \cup T), b^{i}, c \in L^{\infty}(\Omega)$;

- $a^{i j} \zeta_{i} \zeta_{j} \geq \lambda|\zeta|^{2}$, for all $\zeta \in \mathbb{R}^{n}$;

- $\left|a^{i j}\right|,\left|b^{i}\right|,|c| \leq \Lambda$,

where $i, j=1, \ldots, n$. Then, for any domain $\Omega^{\prime} \subset \subset \Omega \cup T$,

$$
\|u\|_{W^{2, p}\left(\Omega^{\prime}\right)} \leq C\left(\|u\|_{L^{p}(\Omega)}+\|f\|_{L^{p}(\Omega)}\right)
$$

where $C$ depends on $n, p, \lambda, \Lambda, T, \Omega^{\prime}, \Omega$, and the moduli of continuity of the coefficients $a^{i, j}$ on $\Omega^{\prime}$ (see Theorem 9.13 [22, p. 239]).

Remark 2.4.3. When $T=\partial \Omega$ in Theorem 2.4.2, we may take $\Omega^{\prime}=\Omega$ to obtain a global $W^{2, p}(\Omega)$ estimate.

The existence of a strong solution for a Dirichlet problem is provided in the following theorem. We refer to [22] for more details.

Theorem 2.4.4 (Existence of a strong solution for Dirichlet problem). Let $\Omega$ be a bounded domain in $\mathbb{R}^{n}$ with $C^{1,1}$ boundary. Let the operator $L$, defined in (2.5), be strictly elliptic in $\Omega$, with coefficients $a^{i j} \in C^{0}(\bar{\Omega}), b^{i}, c \in L^{\infty}$, with $i, j=1, \ldots, n$ and $c \leq 0$. Then, if $f \in L^{p}(\Omega)$ and $\varphi \in W^{2, p}(\Omega)$, with $1<p<\infty$, the Dirichlet problem $L u=f$ in $\Omega, u-\varphi \in W_{0}^{1, p}(\Omega)$ has a unique solution $u \in W^{2, p}(\Omega)$ (see Theorem 9.15 [22, p. 241]).

\subsection{Sub- and Supersolution}

In Chapter 3, we are going to use a vesrion of the sub- and supersolution method for finding a solution for the semilinear elliptic equation. As a result, in this section we bring the definition of sub- and supersolutions for the specific case of (1.1) that we will be considering. 
Definition 2.5.1 (Weak sub- and supersolution). Suppose $\Omega$ is a smooth, bounded domain in $\mathbb{R}^{n}$, and let $g: \Omega \times \mathbb{R} \rightarrow \mathbb{R}$ be a Carathéodory function. Consider the following equation.

$$
\begin{cases}-\Delta u=g(x, u), & \text { in } \Omega, \\ u=0, & \text { on } \partial \Omega .\end{cases}
$$

We say that $u \in H_{0}^{1}(\Omega)$ is a weak subsolution to (2.32) if

$$
\int_{\Omega} \nabla u \nabla \varphi d x-\int_{\Omega} g(x, u) \varphi d x \leq 0 \quad \text { for all } \varphi \text { in } C_{0}^{\infty}(\Omega), \varphi>0
$$

Similarly, $u \in H_{0}^{1}(\Omega)$ is a weak supersolution to (2.32) if in the above the reverse inequality holds. (see [36, p.16]).

\subsection{Weak Maximum principle}

Maximum principle is a useful tool in estimating the solution of second order elliptic equations. Particularly, looking for a solution of a PDE in a convex set constructed by weak sub- and supersolutions, the maximum principle can provide us with the needed estimate for showing the existence of such a solution.

In order to apply the maximum principle for a weak solution, we consider the equation (2.3) in the divergence form (2.4). We say a function $u \in H_{0}^{1}(\Omega)$ satisfies $L u \geq 0(\leq 0)$ in the weak sense, if $B[u, v] \leq 0(\geq 0)$ for all $v \in H_{0}^{1}(\Omega)$, where $B[u, v]$ is defined in (2.11). We can now bring the statement of maximum principle in the weak sense as in [22].

Theorem 2.6.1 (The weak maximum principle). Let $u \in H^{1}(\Omega)$ satisfy $L u \geq$ $0(\leq 0)$ in $\Omega$ in the weak sense. Then

$$
\sup _{\Omega} u \leq \sup _{\partial \Omega} u^{+} \quad\left(\inf _{\Omega} u \geq \inf _{\partial \Omega} u^{-}\right) .
$$

(See Theorem 8.1 [22, page.179]). 
Note that a function $u \in H^{1}(\Omega)$, is said to satisfy $u \leq 0$ on $\partial \Omega$ if $u^{+}=$ $\max \{u, 0\} \in H_{0}^{1}(\Omega)$.

\subsection{Convex Analysis}

In order to define the functional $I[\cdot]$, introduced in the introduction, and apply the variational method, it is essential to review some fundamental definitions and results from convex analysis for lower semi-continuous functions. Therefore, we devote this section to a few of these important definitions and results which will be used frequently in the next sections.

Consider the duality pairing $\left(V, V^{*},\langle\cdot, \cdot\rangle\right)$, that is a real Banach spaces $V$ and its topological dual $V^{*}$, and a bilinear map $\left(u, u^{*}\right) \rightarrow\left\langle u, u^{*}\right\rangle$ into $\mathbb{R}$ which separates points:

$$
\begin{array}{ll}
\forall u \in V, \quad \exists u^{*} \in V^{*}: & \left\langle u, u^{*}\right\rangle \neq 0, \\
\forall u^{*} \in V^{*}, \quad \exists u \in V: & \left\langle u, u^{*}\right\rangle \neq 0 .
\end{array}
$$

Let $F: V \rightarrow \mathbb{R} \cup\{+\infty\}$. We define its domain to be the set of points $u \in V$ where $F(u)$ is finite,

$$
\operatorname{Dom}(F):=\{u \in V \mid F(u)<+\infty\} .
$$

We shall say that $F$ is proper if it is not identically $+\infty$ (see [19, p.79] for more details).

Definition 2.7.1 (Weak lower semi-continuity). A function $F: V \rightarrow \mathbb{R} \cup\{+\infty\}$ is called weakly lower semi-continuous if

$$
F(u) \leq \liminf _{n \rightarrow \infty} F\left(u_{n}\right),
$$

for each $u \in V$ and any sequence $\left\{u_{n}\right\}$ converging weakly to $u$. 
Definition 2.7.2 (The sub-differential of a convex function). Consider the proper convex function $F: V \rightarrow(-\infty,+\infty]$. The sub-differential of $F$ is the mapping $\partial F: V \rightarrow 2^{V^{*}}$ defined by

$$
\partial F(u)=\left\{u^{*} \in V^{*} ; F(u)-F(v) \leq\left\langle u-v, u^{*}\right\rangle, \forall v \in V\right\} .
$$

The elements $u^{*} \in \partial F(u)$ are called sub-gradients of $F$ at $u$. The set of those $u$ for which $\partial F(u) \neq \emptyset$ is called the domain of $\partial F$ and is denoted by $\operatorname{Dom}(\partial F)$. Clearly, if $F$ is not the constant $+\infty, \operatorname{Dom}(\partial F)$ is a subset of $\operatorname{Dom}(F)$. The function $F$ is said to be sub-differentiable at $u$, if $u \in \operatorname{Dom}(\partial F)$ (see [6, p.82] for more details).

The following definitions and results are from $[19,20]$ where the proofs can be found.

Definition 2.7.3 (Gâteaux differentiability). A function $F: V \rightarrow \mathbb{R} \cup\{+\infty\}$, finite in a neighborhood of $u$, is Gâteaux differentiable at $u$ if there exists some $u^{*} \in V^{*}$ such that:

$$
\forall v \in V, \quad \lim _{h \rightarrow 0} \frac{F(u+h v)-F(u)}{h}=\left\langle u^{*}, v\right\rangle .
$$

We then write $D F(u)=u^{*}$.

When $F$ is lower semi continuous, convex and finite on some neighborhood of $u \in V$, it must be continuous at $u$, and hence, sub-differentiable at $u$, but it need not be differentiable in any sense. So, sub-differentiability is a strict extension of differentiability, as the following result shows:

Proposition 2.7.1. If $F: V \rightarrow(-\infty,+\infty]$ is a convex and lower semi-continuous function and if its sub-differential at $u$ is a singleton, $\partial F(u)=\left\{u^{*}\right\}$, then $F$ is Gâteaux differentiable at $u$ and $u^{*}=D F(u)$. If $F: V \rightarrow(-\infty,+\infty]$ is Gâteaux differentiable and continuous at $u$, then $\partial F(u)=\{D F(u)\}$ (see Proposition 9, [19, p.92] for more details). 
We define the Fenchel dual of a function as below.

Definition 2.7.4 (Fenchel dual). Let $F: V \rightarrow \mathbb{R} \cup\{+\infty\}$ be a proper function. The function $F^{*}: V^{*} \rightarrow \mathbb{R} \cup\{+\infty\}$ defined by

$$
F^{*}\left(u^{*}\right)=\sup _{u \in V}\left\{\left\langle u, u^{*}\right\rangle-F(u)\right\}
$$

is called the Fenchel dual or Legendre transform of $F$.

As an easy consequence of Definition (2.7.4), we have Fenchel's inequality,

$$
F(u)+F^{*}\left(u^{*}\right) \geq\left\langle u, u^{*}\right\rangle, \quad \forall u \in V, \forall u^{*} \in V^{*} .
$$

Proposition 2.7.2. If $F$ is a convex and lower semi-continuous function, then $\left(F^{*}\right)^{*}=F$ and the three properties are equivalent:

1. $u^{*} \in \partial F(u)$,

2. $u \in \partial F^{*}\left(u^{*}\right)$,

3. $F(u)+F^{*}\left(u^{*}\right)=\left\langle u, u^{*}\right\rangle$.

Theorem 2.7.5. Let $\Omega$ be a bounded open domain in $\mathbb{R}^{n}$ and $f$ a nonnegative Carathéodory function on $\Omega \times \mathbb{R}$. Put the spaces $L^{p}(\Omega)$ with $1 \leq p \leq \infty$, in duality with $L^{p^{\prime}}(\Omega)$, i.e. $1 / p+1 / p^{\prime}=1$, and define a functional on $L^{p}(\Omega)$, by

$$
F(u)=\int_{\Omega} f(x, u(x)) d x
$$

whence its Fenchel dual $F^{*}$ on $L^{p^{\prime}}(\Omega)$ is

$$
\begin{aligned}
F^{*}\left(u^{*}\right) & =\sup _{u \in L^{p}(\Omega)}\left\{\left\langle u, u^{*}\right\rangle-\int_{\Omega} f(x, u(x)) d x\right\} \\
& =\sup _{u \in L^{p}(\Omega)}\left\{\int_{\Omega}\left[u(x) u^{*}(x)-f(x, u(x))\right] d x\right\} .
\end{aligned}
$$

Now assume that there exists $u_{0} \in L^{\infty}(\Omega)$ such that $F\left(u_{0}\right)<\infty$. Then for all $u^{*} \in L^{p^{\prime}}(\Omega)$, we have 


$$
F^{*}\left(u^{*}\right)=\int_{\Omega} f^{*}\left(x ; u^{*}(x)\right) d x,
$$

where

$$
f^{*}(x, s)=\sup _{t \in \mathbb{R}}\{t s-f(x, t)\} .
$$

(See Proposition 2.1 [20, p.271] for a proof).

Having the required concepts and notation properly defined, we can introduce the new variational principle introduced in [26] in the next chapter. 


\section{Chapter 3}

\section{Variational Problems}

Many partial differential equations are of the form

$$
\Lambda[u]=0,
$$

where $\Lambda: V \rightarrow V^{*}$ denotes a partial differential operator, possibly nonlinear, from an appropriate reflexive Banach space $V$ to its topological dual $V^{*}$, with the bilinear duality pairing $\langle\cdot, \cdot\rangle$ between them. When the problem is variational, there exists a functional $I: V \rightarrow \mathbb{R}$ for which solutions of the PDE (3.1) correspond to critical points of $I[\cdot]$. Assume that the desired functional $I[\cdot]$ is differentiable, with a Gâteaux derivative $D I[\cdot]$. Then, by definition its critical points satisfy

$$
\langle D I[u], v\rangle=\lim _{t \rightarrow 0} \frac{I[u+t v]-I[u]}{t}=0 \quad \text { for all } v \in V .
$$

Hence, for our problem to be variational we should have $\Lambda[u]=D I[u]$. In this case we call $I[\cdot]$ the Euler-Lagrange functional, and critical points of $I[\cdot]$ are weak solutions of this equation (3.1). Beside this functional, other types of functionals have been applied by other authors in the literature. In [26], the author has established a new class of functionals with this desired property. In this approach, he considers a specific kind of partial differential equations which can be written as

$$
A[u]=D \varphi(u), \quad u \in V,
$$


where $A: \operatorname{Dom}(A) \subset V \rightarrow V^{*}$ is a linear operator and $\varphi: V \rightarrow \mathbb{R}$ is a convex, Gâteaux differentiable and lower semi-continuous function. It can be easily seen that for an equation in this form, the Euler-Lagrange functional is $I[u]=\frac{1}{2}\langle A u, u\rangle-\varphi(u)$.

Functionals proposed in [26], need not necessarily be Gâteaux differentiable, so in order to set up the problem in a variational form, a more general definition of critical points is needed. As a result, he defines a critical point for the general form of new functionals as follows.

Definition 3.0.6. Let $\Psi: V^{*} \rightarrow \mathbb{R} \cup\{+\infty\}$ be a convex and lower semicontinuous function and let $F: V \rightarrow \mathbb{R}$ be Gâteaux differentiable. Assume that $A: \operatorname{Dom}(A) \subset V \rightarrow V^{*}$ is a linear operator and $\lambda \in \mathbb{R}$ is a scalar. We call $u \in V$ a critical point of

$$
I(w):=\Psi(A w)+F(w)+\lambda\langle A w, w\rangle,
$$

if $I(u)$ is finite and there exists $v \in \partial \Psi(A u)$ such that

$$
\langle v, A \eta\rangle+\langle D F(u), \eta\rangle+\lambda\langle A \eta, u\rangle+\lambda\langle A u, \eta\rangle=0, \quad \text { for all } \eta \in \operatorname{Dom}(A)
$$

Applying this definition and using the theory of non-convex self-duality, in [26] the author has broadened the functionals for which one can associate their critical points to the solution of the equation (3.2). These functionals and their variational properties are stated and proved in the following theorem (see [26] for the proof).

Theorem 3.0.7. Let $V$ be a reflexive Banach space and $V^{*}$ its topological dual. Let $\varphi: V \rightarrow \mathbb{R}$ be a Gâteaux differentiable convex and lower semi-continuous function, with $\varphi^{*}$ its Fenchel dual. Furthermore, let $A: \operatorname{Dom}(A) \subset V \rightarrow V^{*}$ be a surjective linear operator such that its domain is dense in $V$. For each $\alpha, \beta \in \mathbb{R}$ and $u \in V$ define

$$
I_{\alpha, \beta}(u):=\beta \varphi^{*}(A u)-\alpha \varphi(u)-\frac{\beta-\alpha}{2}\langle A u, u\rangle .
$$


Then, critical points of $I_{\alpha, \beta}($.$) are solutions of the equation$

$$
A u=D \varphi(u),
$$

provided either of the following conditions hold

1. A is a symmetric operator, $\alpha \neq 0$ and $\beta=0$.

2. $A$ is a symmetric operator, $\alpha=0$ and $\beta \neq 0$.

3. $A$ is a negative operator, $\beta=-\alpha \neq 0$.

4. $A$ is a positive symmetric operator and $\alpha \beta>0$.

5. A is a negative symmetric operator and $\alpha \beta<0$.

As one can see in Theorem 3.0.7, by choosing different values for $\alpha, \beta$, various variational principles can be found including the classical ones. For instance, by setting $\alpha=1$, and $\beta=0$ we obtain $I[u]=\frac{1}{2}\langle A u, u\rangle-\varphi(u)$ which is the well-known Euler-Lagrange functional corresponding to the equation.

Although for many equations these functionals are more practical than the Euler-Lagrange functional, finding the critical point on the whole space $V$ will cause some difficulties especially when the problem is supercritical and we lack compactness. To overcome this challenge, based on one of the functionals introduced in Theorem 3.0.7, with $\beta=1, \alpha=1$, in [26] the author also introduced a new variational principle. In this principle, he proved upon fulfillment of appropriate assumptions, a solution for equation (3.1) corresponds to the critical point of the associated functional on a closed and convex subset of the space $V$. To clarify this interesting and useful result on which this thesis is based, we devote the next section to the introduction of this new principle. 


\subsection{A New Variational Principle}

Using the definitions and result from the previous sections, we give a short introduction to the variational principle which is adapted from [13].

Let $V$ be a reflexive Banach space, $V^{*}$ be its topological dual and $K$ be a closed convex subset of $V$. Assume that $\varphi: V \rightarrow \mathbb{R}$ is convex, Gâteaux differentiable, with Gâteaux derivative $D \varphi(\cdot)$, and lower semi-continuous and that $A: V \rightarrow V^{*}$ is a positive linear symmetric operator. Let $\varphi^{*}$ be the Fenchel dual of $\varphi$, i.e.

$$
\varphi^{*}\left(u^{*}\right)=\sup \left\{\left\langle u^{*}, u\right\rangle-\varphi(u) ; u \in V\right\}, \quad u^{*} \in V^{*}
$$

where the pairing between $V$ and $V^{*}$ is denoted by $\langle\cdot, \cdot\rangle$. Define the function $\psi_{K}: V \rightarrow(-\infty,+\infty]$ by

$$
\psi_{K}(u)= \begin{cases}\varphi^{*}(A u), & u \in K, \\ +\infty, & u \notin K,\end{cases}
$$

and set $\operatorname{Dom}\left(\psi_{K}\right)=\left\{u \in V ; \psi_{K}(u)<\infty\right\}$. Consider the functional $I_{K}: V \rightarrow$ $(-\infty,+\infty]$ defined by

$$
I_{K}[w]:=\psi_{K}(w)-\varphi(w)
$$

As in [37], a point $u \in \operatorname{Dom}\left(\psi_{K}\right)$ is said to be a critical point of $I_{K}$ if

$$
\psi_{K}(v)-\psi_{K}(u) \geq\langle D \varphi(u), v-u\rangle, \quad \forall v \in V .
$$

We shall now recall the following variational principle established in [26].

Theorem 3.1.1. Let $V$ be a reflexive Banach space and $K$ be a closed convex subset of $V$. Let $\varphi: V \rightarrow \mathbb{R}$ be a Gâteaux differentiable convex and lower semicontinuous function, and let the linear operator $A: \operatorname{Dom}(A) \subset V \rightarrow V^{*}$ be symmetric and positive. Assume that $u$ is a critical point of $I_{K}(w)=\psi_{K}(w)-$ $\varphi(w)$, and that there exists $v \in K$ satisfying the linear equation,

$$
A v=D \varphi(u)
$$


Then $u \in K$ is a solution of the equation,

$$
A u=D \varphi(u) .
$$

This new variational principle is the basis for this thesis and has the main contribution in the establishment of our results. In Chapter 4 and Chapter 5 , we apply this principle as a main tool to prove the existence of solutions for particular types of equations (1.1).

\subsection{Applying the New Variational Principle}

To apply the new principle, we need to state and prove the adapted version of Theorem 3.1.1 to our case. In order to do that, first we set up $V, K, \varphi$ and $\psi$ accordingly.

Recall the general form of equation (1.1) which we are considering in this work. We assume that $g: \Omega \times \mathbb{R} \rightarrow \mathbb{R}$ is a Carethéodory function which is increasing in $u$ and satisfies a growth condition of the form

$$
|g(x, u)| \leq a(x)\left(|u|^{p-1}+1\right)
$$

where $0<a(x) \in L^{\infty}(\Omega)$ and $p>2$ is a real number. Furthermore, we set $b(x) \in L^{2}(\Omega)$. Now, set $V$ to be the Banach space $H_{0}^{1}(\Omega) \cap L^{p}(\Omega)$ with the norm

$$
\|u\|_{V}:=\|u\|_{H_{0}^{1}(\Omega)}+\|u\|_{L^{p}(\Omega)} .
$$

Let $V^{*}$ be the topological dual of $V$, and denote by $\langle\cdot, \cdot\rangle$ the pairing between $V$ and $V^{*}$ which is defined by

$$
\left\langle v, v^{*}\right\rangle=\int v(x) v^{*}(x) d x, \quad \forall v \in V, \forall v^{*} \in V^{*}
$$

For $v \in V$ define the linear operator $A: \operatorname{Dom}(A) \subset V \rightarrow V^{*}$ by $A v:=-\Delta v+\lambda v$, with $\lambda \geq 0$. It can be easily proved that $A$ satisfies the assumptions of Theorem 3.1.1. 
Lemma 3.2.1. The linear operator $A: \operatorname{Dom}(A) \subset V \rightarrow V^{*}$ defined by $A v:=$ $-\Delta v+\lambda v$, with $\lambda \geq 0$, is symmetric and positive.

Proof. Recalling Definition 2.2.11, to show that $A$ is symmetric we need to show $\langle A u, v\rangle=\langle u, A v\rangle$ for all $u, v \in \operatorname{Dom}(A)$. Following the definition of $A$, and using integration by part we find

$$
\begin{aligned}
\langle A u, v\rangle & =\int_{\Omega}(-\Delta u v+\lambda u v) d x \\
& =\int_{\Omega}(-\Delta v u+\lambda v u) d x=\langle u, A v\rangle,
\end{aligned}
$$

as desired. Next, we claim that $A$ is positive. Indeed, by an easy calculation we find

$$
\begin{aligned}
\langle A u, u\rangle & =\int_{\Omega}\left(-\Delta u u+\lambda u^{2}\right) d x \\
& =\int_{\Omega}\left(|\nabla u|^{2}+\lambda|u|^{2}\right) d x,
\end{aligned}
$$

which is positive as by assumption we have $\lambda \geq 0$.

Next, set $G: \Omega \times \mathbb{R} \rightarrow \mathbb{R}$ to be the primitive of $g(x, \cdot)$, i.e. $G(x, y)=$ $\int_{0}^{y} g(x, s) d s$. Then, we have that

$$
\int_{0}^{y} \mu g(x, s)+b(x) d s=\mu G(x, y)+b(x) y .
$$

Now, define $\varphi: V \rightarrow \mathbb{R}$ by

$$
\varphi(u):=\int_{\Omega} \mu G(x, u)+u b(x) d x .
$$

Before we continue establishing the desired functional, we need to prove that $\varphi$ satisfies the conditions in Theorem 3.1.1.

Lemma 3.2.2. The functional $\varphi(\cdot)$ defined in (3.8) is Gâteaux differentiable, convex and lower semi-continuous. 
Proof. As $g$ satisfies the growth condition (3.7), it is standard that $\varphi(\cdot)$ is of class of $C^{1}$ (see [39] for a proof). Hence, it can be deduced that $\varphi(\cdot)$ is Gatéaux differentiable and continuous and we have

$$
\langle D \varphi(u), v\rangle=\langle\mu g(x, u)+b(x), v\rangle .
$$

It remains to show that $\varphi(\cdot)$ is convex. First note that as $g(x, \cdot)$ is continuous and increasing with respect to $u$, we can assert that $G(x, \cdot)$ is convex with respect to $u$. Therefore, for $u, v \in V$ and $t \in[0,1]$ we can conclude

$$
\begin{aligned}
\varphi(t u+(1-t) v) & =\int_{\Omega} \mu G(x, t u+(1-t) v)+b(x)(t u+(1-t) v) d x \\
& \leq \int_{\Omega} \mu(t G(x, u)+(1-t) G(x, v)) d x+\int_{\Omega}(t u b(x)+(1-t) v b(x)) d x \\
& =t \int_{\Omega} \mu G(x, u)+u b(x) d x+(1-t) \int_{\Omega} \mu G(x, v)+v b(x) d x \\
& =t \varphi(u)+(1-t) \varphi(v) .
\end{aligned}
$$

which implies the convexity of $\varphi(\cdot)$.

Having $\varphi$ and $A$ appropriately defined, we can rewrite the problem (1.1) as

$$
A u=D \varphi(u)
$$

Let $G^{*}: \Omega \times \mathbb{R} \rightarrow(-\infty,+\infty]$ be the Fenchel dual of $G$, i.e.

$$
G^{*}(x, s)=\sup _{t \in \mathbb{R}}\{t s-G(x, t)\}
$$

Now, we need to find the Fenchel dual of $\varphi(\cdot)$.

Lemma 3.2.3. Consider the functional $\varphi: V \rightarrow \mathbb{R}$ defined in (3.8). Let $\varphi^{*}$ : $V^{*} \rightarrow \mathbb{R}$ be the Fenchel dual of $\varphi$. Then, for each $h \in L^{p^{\prime}}(\Omega)$, with $1 / p+1 / p^{\prime}=1$, we have

$$
\varphi^{*}(h)=\mu \int G^{*}\left(x, \frac{h(x)-b(x)}{\mu}\right) d x .
$$


Proof. Take $h \in L^{p^{\prime}}(\Omega)$. From the definition of Fenchel dual and the density of $V$ in $L^{p}(\Omega)$, we observe that

$$
\begin{aligned}
\varphi^{*}(h) & =\sup _{v \in V}\{\langle v, h\rangle-\varphi(v)\} \\
& =\sup _{v \in V}\left\{\int_{\Omega} v(x) h(x) d x-\int_{\Omega} \mu G(x, v) d x-\int_{\Omega} b(x) v(x) d x\right\} \\
& =\sup _{v \in L^{p}(\Omega)}\left\{\int_{\Omega} v(x) h(x) d x-\int_{\Omega} \mu G(x, v) d x-\int_{\Omega} b(x) v(x) d x\right\} \\
& =\mu \sup _{v \in L^{p}(\Omega)}\left\{\int_{\Omega} v(x) \frac{h(x)-b(x)}{\mu} d x-G(x, v) d x\right\} .
\end{aligned}
$$

Since $G(x, 0)=0$ and $\varphi(0)=0<\infty$, as a consequence of Theorem 2.7.5, we obtain

$$
\varphi^{*}(h)=\int_{\Omega} \mu G^{*}\left(x, \frac{h(x)-b(x)}{\mu}\right) d x .
$$

Now, consider a closed convex subset $K$ of $V$, which depending on each of our problems will be chosen appropriately. We define $\psi(\cdot)$ as follows:

$$
\psi(u):= \begin{cases}\int_{\Omega} \mu G^{*}\left(x, \frac{-\Delta u+\lambda u-b(x)}{\mu}\right) d x, & u \in K \cap W^{2, p^{\prime}}(\Omega), \\ +\infty & u \notin K \cap W^{2, p^{\prime}}(\Omega),\end{cases}
$$

with $\operatorname{Dom}(\psi)=\{u \in V ; \psi(u)<\infty\}$. As $G^{*}$ is a convex function and $K$ is a convex set, it can be easily seen that $\psi$ is also a convex function. It is worth noting that for $u \in \operatorname{Dom}(\psi)$, we have $A u=-\Delta u+\lambda u \in L^{p^{\prime}}(\Omega)$, and hence by Lemma 3.2.3, $\psi(u)=\varphi^{*}(A u)$.

Finally, set

$$
I[u]=\psi(u)-\varphi(u) .
$$

We shall now rephrase and prove Theorem 3.1.1 for our case as follows.

Corollary 3.2.4. Assume that $u$ is a minimum of

$$
I[w]:=\psi(w)-\phi(w),
$$


where $\psi$ and $\phi$ are given in (3.9) and (3.8), respectively. If there exists $v \in$ $\operatorname{Dom}(\psi)$ satisfying the linear equation,

$$
\begin{cases}-\Delta v+\lambda v=\mu g(x, u)+b(x), & \text { in } \Omega, \\ v=0, & \text { on } \partial \Omega,\end{cases}
$$

then $u$ is a solution of the equation,

$$
\begin{cases}-\Delta u+\lambda u=\mu g(x, u)+b(x), & \text { in } \Omega, \\ u=0, & \text { on } \partial \Omega .\end{cases}
$$

Proof. First note that $u$ being a minimum of $I[\cdot]$ implies that $u$ is a critical point of $I[\cdot]$. Indeed, as $\psi$ is convex, we can conclude that for each $w \in V$ and small $t>0$ we have that

$$
\begin{aligned}
0 \leq I[(1-t) u+t w]-I[u] & =\psi((1-t) u+t w)-\varphi((1-t) u+t w)-\psi(u)+\varphi(u) \\
& \leq(1-t) \psi(u)+t \psi(w)-\varphi((1-t) u+t w)-\psi(u)+\varphi(u) \\
& =t(\psi(w)-\psi(u))-\varphi((1-t) u+t w)+\varphi(u) .
\end{aligned}
$$

Now if we divide (3.13) by $t$ and take the limit when $t$ approaches $0^{+}$, by definition of the Gâteaux derivative we obtain

$$
\psi(w)-\psi(u) \geq \lim _{t \rightarrow 0^{+}} \frac{\varphi(u+t(w-u))-\varphi(u)}{t}=\langle D \varphi(u), w-u\rangle .
$$

Hence

$$
\psi(w)-\psi(u) \geq\langle D \varphi(u), w-u\rangle, \quad \forall w \in V
$$

which by definition means that $u$ is a critical point of $I[\cdot]$.

As $I[u]$ is finite, we have that $u \in \operatorname{Dom}(\psi)$, and

$$
\psi(u)=\int_{\Omega} \mu G^{*}\left(x, \frac{-\Delta u+\lambda u-b(x)}{\mu}\right) d x<\infty .
$$

In addition, for $u \in \operatorname{Dom}(\psi)$, obviously we have $A u=-\Delta u+\lambda u \in L^{p^{\prime}}(\Omega)$ and therefore $\psi(u)=\varphi^{*}(A u)$. By assumption, $A v=D \varphi(u)$ for $v \in \operatorname{Dom}(\psi)$, so we can substitute $w=v$ in (3.14) to find that

$$
\varphi^{*}(A v)-\varphi^{*}(A u)=\psi(v)-\psi(u) \geq\langle D \varphi(u), v-u\rangle=\langle A v, v-u\rangle .
$$


Furthermore, from Proposition 2.7.2, $A v=D \varphi(u)$ yields $u \in \partial \varphi^{*}(A v)$, so by definition we get

$$
\varphi^{*}(A u)-\varphi^{*}(A v) \geq\langle u, A u-A v\rangle .
$$

Adding (3.15) to (3.16) implies that

$$
\langle u, A u-A v\rangle+\langle A v, v-u\rangle \leq 0 .
$$

Note that $A$ is symmetric, so applying the property of a symmetric operator, we get

$$
\langle u-v, A u-A v\rangle \leq 0,
$$

which means that

$$
\int_{\Omega}|\nabla u-\nabla v|^{2} d x+\lambda \int_{\Omega}|u-v|^{2} d x \leq 0,
$$

which clearly forces $u=v$. It then follows that $A u=A v=D \phi(u)$ as desired.

Now that we have completely adjusted the new variational principle to our problems, we can start proving the main results of this thesis. Note that based on Corollary 3.2.4, the proof of the existence of solutions for equation (1.1) can be broken down into two main steps; first to show that the functional $I[\cdot]$ defined in (3.10) has a minimizer, and second to prove the linear equation (3.12) has a solution in $K$. In the following chapters, we verify these two steps for each of our problems by applying appropriate preliminary results of Chapter 2 . 


\section{Chapter 4}

\section{Sub- and Supersolutions}

\subsection{Introduction}

In this chapter, we present a variational sub-supersolution method via the new principle introduced in $[25,26]$ to prove the existence of solutions for a particular case of equation (1.1), which is of the following form

$$
\left\{\begin{aligned}
-\Delta u & =g(x, u), & & \text { in } \Omega, \\
u & =0, & & \text { on } \partial \Omega,
\end{aligned}\right.
$$

where $\Omega$ is a bounded domain in $\mathbb{R}^{n}$ and $g: \Omega \times \mathbb{R} \rightarrow \mathbb{R}$ is a Carathéodory function.

From the basic method of sub- and supersolutions (see [34]), it is well-known that if $g$ is smooth and if there exist smooth sub and supersolutions $\underline{u}_{1} \leq \bar{u}_{2}$ of (4.1), then problem (4.1) admits a classical solution $u$, where $\underline{u}_{1} \leq u \leq \bar{u}_{2}$. To prove this via a monotone iteration scheme, which is the common approach for this problem, $g$ needs to be Lipschitz. In [14], by applying Schauder's fixed point theorem along with a version of the strong maximum principle, Clement and Sweers proved that (4.1) admits a solution if sub- and supersolutions $\underline{u}_{1}$ and $\bar{u}_{2}$, defined in the weak sense, are in $C(\bar{\Omega})$, and $g: \Omega \times \mathbb{R} \rightarrow \mathbb{R}$ is continuous. 
When weak sub- and supersolutions $\underline{u}_{1}, \bar{u}_{2} \in H^{1}(\Omega)$, Dancer and Sweers in [15] have shown the existence of a solution $u \in H_{0}^{1}(\Omega)$ for problem (4.1) when $g$ : $\Omega \times \mathbb{R} \rightarrow \mathbb{R}$ is a Carathéodory function and $\sup \left\{g(x, v) ; \underline{u}_{1} \leq v \leq \bar{u}_{2}\right\} \in L^{p}(\Omega)$ with $p>2 n / n+2$. Similar results have been found by Montenegro and Ponce in [27], where they used an extended version of the sub- and supersolution method based on Schauder's fixed point theorem and proved the existence $u \in H_{0}^{1}(\Omega)$, when $\underline{u}_{1}, \bar{u}_{2} \in L^{1}(\Omega)$ and $g(x, v) \in L^{2 n / n+2}(\Omega)$ for every $v \in L^{1}(\Omega)$ such that $\underline{u}_{1} \leq v \leq \bar{u}_{2}$. Assuming $\underline{u}_{1}, \bar{u}_{2} \in C^{2}(\Omega) \cap H_{0}^{1}(\Omega)$, Struwe used a variational method in [36] to prove the existence of solutions for (4.1) when the nonlinearity $g$ does not have supercritical growth.

Here, we apply a variational approach via the new principle to solve the equation (4.1) when it admits weak sub- and supersolutions $\underline{u}_{1}, \bar{u}_{2} \in H_{0}^{1}(\Omega) \cap L^{p}(\Omega)$, with $p>2$. The combination of the new principle and the sub- and supersolution method allows us to find a solution when not only the nonlinearity $g$ is not smooth or Lipschitz, but may also have supercritical growth. The main result of this chapter is stated in the following theorem.

Theorem 4.1.1. Suppose $\Omega$ is a bounded domain in $\mathbb{R}^{n}$ with a $C^{1,1}$ boundary and consider the problem

$$
\left\{\begin{aligned}
-\Delta u & =g(x, u), & & \text { in } \Omega, \\
u & =0, & & \text { on } \partial \Omega,
\end{aligned}\right.
$$

and assume it has a weak subsolution $\underline{u}_{1}$ and a weak supersolution $\bar{u}_{2}$ such that $\underline{u}_{1}, \bar{u}_{2} \in H_{0}^{1}(\Omega) \cap L^{p}(\Omega)$ and $\underline{u}_{1} \leq \bar{u}_{2}$ a.e. in $\Omega$. Furthermore, assume $g:(\Omega \times \mathbb{R}) \rightarrow$ $\mathbb{R}$ is a Caratheodory function which satisfies the following assumptions:

$\mathbf{A}_{1-} u \rightarrow g(x, u)$ is increasing.

$\mathbf{A}_{2-}$ There exists $0<a(x) \in L^{\infty}(\Omega)$ and $p>2$ such that

$$
|g(x, u)| \leq a(x)\left(1+|u|^{p-1}\right) .
$$


$\mathbf{A}_{3^{-}} g\left(x, \underline{u}_{1}\right), g\left(x, \bar{u}_{2}\right) \in L^{\frac{2 n}{n+2}}(\Omega)$

Then, the problem (4.2) admits at least one solution $u$, with $\underline{u}_{1} \leq u \leq \bar{u}_{2}$.

We prove this result by verifying the two steps of Corollary 3.2.4, explained in the last chapter. In order to do that, we first define the closed convex set $K=\left\{u \in H_{0}^{1}(\Omega) \cap L^{p}(\Omega) ; \underline{u}_{1} \leq u \leq \bar{u}_{2}\right\}$, in which we can easily find a critical point $\bar{u}$ due to its boundedness. For the second part of the proof, we assert the existence of a solution $\bar{v}$ for the linear equation $A v=D \varphi(\bar{u})$ by an standard result. Then, we show that it belongs to $K \cap W^{2, p^{\prime}}(\Omega)$ by using the maximum principle (Theorem 2.6.1), and elliptic regularity (Theorem 2.4.2). 


\subsection{Main Result}

We establish the result of this chapter by applying Corollary 3.2.4 to problem (4.1). Following the same approach as in Section 3.2, we first recall the definition of $V, K, \varphi$, and $\psi$ for this particular problem. Consider the Banach space $V=H_{0}^{1}(\Omega) \cap L^{p}(\Omega)$ equipped with the norm

$$
\|u\|_{V}:=\|u\|_{H_{0}^{1}(\Omega)}+\|u\|_{L^{p}(\Omega)} .
$$

Set $V^{*}$ to be the topological dual of $V$, with $\langle.,$.$\rangle the pairing between them, and$ define the operator $A: \operatorname{Dom}(A) \subset V \rightarrow V^{*}$ by $A v:=-\Delta v$ for $v \in V$. For function $g: \Omega \times \mathbb{R} \rightarrow \mathbb{R}$ satisfying assumptions $A_{1}-A_{3}$ of Theorem 4.1.1, let $G: \Omega \times \mathbb{R} \rightarrow \mathbb{R}$ be $G(x, u)=\int_{0}^{u} g(x, s) d s$, and $G^{*}: \Omega \times \mathbb{R} \rightarrow(-\infty,+\infty]$ be the Fenchel dual of $G(x, \cdot)$, i.e. $G^{*}(x, s)=\sup _{t \in \mathbb{R}}\{t s-G(x, t)\}$. We define $\varphi: V \rightarrow \mathbb{R}$ by

$$
\varphi(u)=\int_{\Omega} G(x, u) d x
$$

and rewrite the problem (4.1) as

$$
A u=D \varphi(u)
$$

Now, consider the set $K=\left\{u \in V ; \underline{u}_{1} \leq u \leq \bar{u}_{2}\right\}$. Denote by $p^{\prime}=p /(p-1)$ the conjugate of $p$, and define $\psi(\cdot)$ to be

$$
\psi(u):= \begin{cases}\int_{\Omega} G^{*}(x,-\Delta u) d x, & u \in K \cap W^{2, p^{\prime}}(\Omega) \\ +\infty & u \notin K \cap W^{2, p^{\prime}}(\Omega),\end{cases}
$$

with $\operatorname{Dom}(\psi)=\{u \in V ; \psi(u)<\infty\}$. Note that by Lemma 3.2.3 we have

$$
\varphi^{*}(h)=\int_{\Omega} G^{*}(x, h) d x, \quad \text { for } h \in L^{p^{\prime}}(\Omega),
$$

so we can assert that $\psi(u)=\varphi^{*}(A u)$ since for $u \in \operatorname{Dom}(\psi), A u=-\Delta u \in L^{p^{\prime}}(\Omega)$. Finally, set

$$
I[u]=\psi(u)-\varphi(u) .
$$


Before the proof of main theorem, we state the following lemma which is required in the process of the main proof.

Lemma 4.2.1. Assume that $\varphi: V \rightarrow \mathbb{R}$ is defined by $\varphi(u)=\int_{\Omega} G(x, u) d x$, where $G(x, u)=\int_{0}^{u} g(x, s) d s$ and

$$
|g(x, u)| \leq a(x)\left(1+|u|^{p-1}\right),
$$

for $0<a(x) \in L^{\infty}(\Omega)$ and $p>2$. Let $\varphi^{*}: V^{*} \rightarrow(0,+\infty]$ be the Fenchel dual of $\varphi$. Then, there exist positive constants $C_{5}$ and $C_{6}$ such that

$$
\varphi(h) \geq C_{5}\|h\|_{L^{p^{\prime}((\Omega))}}^{p^{\prime}}-C_{6},
$$

for all $h \in L^{p^{\prime}}(\Omega)$.

Proof. Using the assumptions on $a(x)$ and $g(x, u)$, we can conclude that for a constant $C_{1}>0, G(x, v) \leq C_{1}\left(1+|v|^{p}\right)$. Therefore we have

$$
\varphi(v)=\int_{\Omega} G(x, v) d x \leq C_{1} \int_{\Omega}\left(1+|v|^{p}\right) d x,
$$

for all $v \in L^{p}(\Omega)$. From the definition of the Fenchel dual and the density of $V$ in $L^{p}(\Omega)$, we obtain

$$
\begin{aligned}
\varphi^{*}(h) & =\sup _{v \in V}\{\langle v, h\rangle-\varphi(v)\} \\
& \geq \sup _{v \in V}\left\{\int_{\Omega} v(x) h(x) d x-C_{1} \int_{\Omega}\left(1+|v|^{p}\right) d x\right\} \\
& =\sup _{v \in L^{p}(\Omega)}\left\{\int_{\Omega} v(x) h(x) d x-C_{1} \int_{\Omega}\left(1+|v|^{p}\right) d x\right\} \\
& =C_{5}\|h\|_{L^{p^{\prime}}(\Omega)}^{p^{\prime}}-C_{6},
\end{aligned}
$$

for some constants $C_{5}$ and $C_{6}$.

We now prove the main result of this chapter.

Proof of Theorem 4.1.1. To prove that (4.2) has a solution, using Corollary 3.2.4, first we must show that $I[u]$ has a critical point, which is a minimizer, i.e.

$$
\exists \bar{u} \in V \quad \text { such that } \quad I[\bar{u}]=\inf _{u \in V} I[u] .
$$


It follows from $A_{2}$ that for a constant $C_{1}>0, G(x, u) \leq C_{1}\left(1+|u|^{p}\right)$. Then, for $u \in K$, that is $\underline{u}_{1} \leq u \leq \bar{u}_{2}$, we have

$$
G(x, u) \leq C_{1}\left(1+|u|^{p}\right) \leq C_{2}\left(1+\left|\bar{u}_{2}\right|^{p}+\left|\underline{u}_{1}\right|^{p}\right),
$$

for an appropriate constant $C_{2}$. Then, by definition we obtain

$$
\varphi(u)=\int_{\Omega} G(x, u) d x \leq C_{2} \int_{\Omega}\left(1+\left|\bar{u}_{2}\right|^{p}+\left|\underline{u}_{1}\right|^{p}\right) d x
$$

which is finite as $\bar{u}_{2}, \underline{u}_{1} \in L^{p}(\Omega)$. Thus,

$$
I[u]=\varphi^{*}(A u)-\varphi(u) \geq \varphi^{*}(A u)-C_{2} \int_{\Omega}\left(1+\left|\bar{u}_{2}\right|^{p}+\left|\underline{u}_{1}\right|^{p}\right) d x .
$$

So, as $\inf _{u \in K} I[u]>-\infty$, we can consider a minimizing sequence in $K$ i.e.

$$
\left\{u_{n}\right\} \subset K ; \quad \text { such that } \quad I\left[u_{n}\right] \rightarrow \inf _{u \in K} I[u]:=m .
$$

Now we show that $I[\cdot]$ attains its minimum in $\operatorname{Dom}(\psi)$. In order to prove this, first we need to show that $I[\cdot]$ is weakly lower semi-continuous. Again, consider the minimizing sequence $\left\{u_{n}\right\}$. By definition we have

$$
\begin{aligned}
I\left[u_{n}\right] & \geq\left\langle A u_{n}, u_{n}\right\rangle-2 \varphi\left(u_{n}\right) \\
& =\left\|\nabla u_{n}\right\|_{L^{2}(\Omega)}^{2}-2 \varphi\left(u_{n}\right) \\
& =\left\|u_{n}\right\|_{H_{0}^{1}}^{2}-2 \varphi\left(u_{n}\right) \\
& \geq\left\|u_{n}\right\|_{H_{0}^{1}}^{2}-2 C_{2} \int_{\Omega}\left(1+\left|\underline{u}_{1}\right|^{p}+\left|\bar{u}_{2}\right|^{p}\right) d x .
\end{aligned}
$$

As $\underline{u}_{1}, \bar{u}_{2} \in L^{p}(\Omega)$ and $\Omega$ is bounded, we can assert that $I\left[u_{n}\right] \geq\left\|u_{n}\right\|_{H_{0}^{1}(\Omega)}^{2}-C_{3}$ for an appropriate $C_{3}$. Hence, recalling that $I\left[u_{n}\right] \rightarrow m<\infty$ we obtain

$$
\left\|u_{n}\right\|_{H_{0}^{1}(\Omega)}^{2} \leq I\left[u_{n}\right]+C_{3}<\infty
$$

which means that $\left\{u_{n}\right\}$ is bounded in $H_{0}^{1}(\Omega)$. In addition, we can also assert that

$$
\int_{\Omega}\left|u_{n}\right|^{p} d x \leq C_{4}\left(\int_{\Omega}\left|\underline{u}_{1}\right|^{p} d x+\int_{\Omega}\left|\bar{u}_{2}\right|^{p} d x\right)<\infty
$$


which means that $\left\{u_{n}\right\}$ is bounded in $L^{p}(\Omega)$.

As a result, we can conclude that $\left\|u_{n}\right\|_{V}=\left\|u_{n}\right\|_{H_{0}^{1}(\Omega)}+\left\|u_{n}\right\|_{L^{p}(\Omega)}$ is bounded. Since $V$ is reflexive, after passing to a subsequence if necessary, we can assume there exists $\bar{u} \in V$ such that $u_{n} \rightarrow \bar{u}$ in $V$. It follows that $\bar{u} \in K$ since for all $n$ we have $\underline{u}_{1} \leq u_{n} \leq \bar{u}_{2}$, and $K$ is a closed and convex set. Recall that for $u \in \operatorname{Dom}(\psi), A u=-\Delta u \in L^{p^{\prime}}(\Omega)$ and $\psi(u)=\varphi^{*}(A u)$. Hence, as shown in Lemma 4.2.1 we obtain

$$
\psi\left(u_{n}\right)=\varphi^{*}\left(-\Delta u_{n}\right) \geq C_{5}\left\|\Delta u_{n}\right\|_{L^{p^{\prime}}}^{p^{\prime}}(\Omega)-C_{6},
$$

for appropriate constants $C_{5}$ and $C_{6}$. Therefore, having $\sup _{n}\left\{\psi\left(u_{n}\right)\right\}<\infty$, we can conclude that $\left\{u_{n}\right\}$ is bounded in $W^{2, p^{\prime}}(\Omega)$, so up to a subsequence we must have that $u_{n} \rightarrow \bar{u}$ in $W^{2, p^{\prime}}(\Omega)$ and hence $\bar{u} \in K \cap W^{2, p^{\prime}}(\Omega)$.

Now, we claim that $\psi(\cdot)$ is weakly lower semi-continuous. Take $v \in L^{p}(\Omega)$. By definition of $\varphi^{*}\left(-\Delta u_{n}\right)$, we have that

$$
\psi\left(u_{n}\right)=\varphi^{*}\left(-\Delta u_{n}\right) \geq \int_{\Omega} v(x)\left(-\Delta u_{n}\right) d x-\varphi(v),
$$

from which we obtain

$$
\liminf _{n \rightarrow \infty} \psi\left(u_{n}\right)=\liminf _{n \rightarrow \infty} \varphi^{*}\left(-\Delta u_{n}\right) \geq \int_{\Omega} v(x)(-\Delta \bar{u}) d x-\varphi(v) .
$$

Taking sup over all $v \in L^{p}(\Omega)$ implies that

$$
\liminf _{n \rightarrow \infty} \psi\left(u_{n}\right)=\liminf _{n \rightarrow \infty} \varphi^{*}\left(-\Delta u_{n}\right) \geq \varphi^{*}(-\Delta \bar{u})=\psi(\bar{u})
$$

which means that $\psi(\cdot)$ is weakly lower semi-continuous.

In addition, we can prove that $\varphi(\cdot)$ is continuous. Note that as $u_{n} \rightarrow \bar{u}$ in $H_{0}^{1}(\Omega)$, it follows that $u_{n} \rightarrow \bar{u}$ strongly in $L^{2}(\Omega)$, so going if necessary to a subsequence, we have that $u_{n} \rightarrow \bar{u}$ a.e. in $\Omega$. Being the primitive of a Carathéordory function, $G(x, \cdot)$ is continuous, so we can deduce that $G\left(x, u_{n}\right) \rightarrow G(x, \bar{u})$ for a.e. $x \in \Omega$. Furthermore, recalling that $G\left(x, u_{n}\right) \leq C_{1}\left(1+\left|u_{n}\right|^{p}\right) \leq C_{2}\left(1+\left|\bar{u}_{2}\right|^{p}+\left|\underline{u}_{1}\right|^{p}\right)$, 
and $\underline{u}_{1}, \bar{u}_{2} \in L^{p}(\Omega)$, by Lebesgue dominated converges theorem we deduce

$$
\lim _{n \rightarrow \infty} \int_{\Omega} G\left(x, u_{n}\right) d x=\int_{\Omega} \lim _{n \rightarrow \infty} G\left(x, u_{n}\right) d x=\int_{\Omega} G(x, \bar{u}) d x,
$$

and hence $\varphi\left(u_{n}\right) \rightarrow \varphi(\bar{u})$, which means that $\varphi(\cdot)$ is continuous.

Now, by definition we have

$$
\liminf _{n \rightarrow \infty} I\left[u_{n}\right]=\liminf _{u \rightarrow \infty}\left(\psi\left(u_{n}\right)-\varphi\left(u_{n}\right)\right)
$$

and by continuity of $\varphi(\cdot)$ we obtain

$$
\liminf _{n \rightarrow \infty} I\left[u_{n}\right]=\liminf _{n \rightarrow \infty} \psi\left(u_{n}\right)-\lim _{n \rightarrow \infty} \varphi\left(u_{n}\right) \geq \psi(\bar{u})-\varphi(\bar{u})=I[\bar{u}],
$$

which means that $I[\cdot]$ is lower semi-continuous. Therefore, as $u_{n} \rightarrow \bar{u}$ in $K \cap$ $W^{2, p^{\prime}}(\Omega)$, we obtain

$$
m \leq I[\bar{u}] \leq \liminf _{n \rightarrow \infty} I\left[u_{n}\right]=m,
$$

which implies that $\bar{u}$ is a minimizer of $I[\cdot]$.

Following Corollary 3.2.4, to show the existence of a solution for (4.2), in the next step, we need to prove that there exists a $\bar{v} \in \operatorname{Dom}(\psi)$ satisfying

$$
\left\{\begin{aligned}
-\Delta v & =g(x, \bar{u}), & & \text { in } \Omega, \\
v & =0, & & \text { on } \partial \Omega,
\end{aligned}\right.
$$

First we show that there exists a $\bar{v} \in H_{0}^{1}(\Omega)$ which satisfies (4.5). Note that $g(x, \bar{u}) \in L^{\frac{2 n}{n+2}}(\Omega)$. Indeed, by assumption $A_{3}$ we have $g\left(x, \underline{u}_{1}\right), g\left(x, \bar{u}_{2}\right) \in L^{\frac{2 n}{n+2}}(\Omega)$. Hence, by using $A_{1}$ and $A_{3}$ we obtain

$$
\|g(x, \bar{u})\|_{L^{\frac{2 n}{n+2}(\Omega)}} \leq C_{6}\left(\left\|g\left(x, \underline{u}_{1}\right)\right\|_{L^{\frac{2 n}{n+2}(\Omega)}}+\left\|g\left(x, \bar{u}_{2}\right)\right\|_{L^{\frac{2 n}{n+2}}(\Omega)}\right)
$$

which means that $g(x, \bar{u}) \in L^{\frac{2 n}{n+2}}(\Omega)$.

Now, consider the Euler-Lagrange energy functional for (4.5)

$$
E(v)=\frac{1}{2} \int_{\Omega}|\nabla v|^{2} d x-\int_{\Omega} g(x, \bar{u}) v d x
$$


which is well-defined for $v \in H_{0}^{1}(\Omega)$ as $g(x, \bar{u}) \in L^{\frac{2 n}{n+2}}$ and $H_{0}^{1}(\Omega) \hookrightarrow L^{2^{*}}(\Omega)$, where $2^{*}=\frac{2 n}{n-2}$ for $n \geq 3$ and $2^{*}=\infty$ for $n=1,2$. Set

$$
E(\bar{v})=\inf _{v \in V} E(v)
$$

then, $\bar{v}$ is a critical point of $E(v)$ and satisfies (4.5).

Furthermore, we must show that $\bar{v} \in K$. Note that for $u \in K$ we have $\underline{u}_{1} \leq u \leq \bar{u}_{2}$. Now recalling the facts that $\bar{v}$ is a weak solution of (4.5), $\bar{u}_{2}$ is a weak supersolution of $(4.2)$ and $g(x, \cdot)$ is an increasing function of $u$, for any non-negative $w \in H_{0}^{1}(\Omega)$ we obtain

$$
\int_{\Omega} \nabla \bar{v} \cdot \nabla w d x=\int_{\Omega} g(x, \bar{u}) w d x \leq \int_{\Omega} g\left(x, \bar{u}_{2}\right) w d x \leq \int_{\Omega} \nabla \bar{u}_{2} \cdot \nabla w d x .
$$

In addition, by assumption we also have

$$
\bar{v}=0 \quad \text { and } \quad \bar{u}_{2}=0, \text { on } \partial \Omega,
$$

in the sense that $\bar{v}, \bar{u}_{2} \in H_{0}^{1}(\Omega)$, from which we can conclude that

$$
\bar{v}-\bar{u}_{2} \in H_{0}^{1}(\Omega)
$$

Note that by a standard result in Sobolev spaces, (4.7) implies that $\left(\bar{v}-\bar{u}_{2}\right)^{+} \in$ $H_{0}^{1}(\Omega)$ as well. Hence, form (4.6) and (4.7) we see that

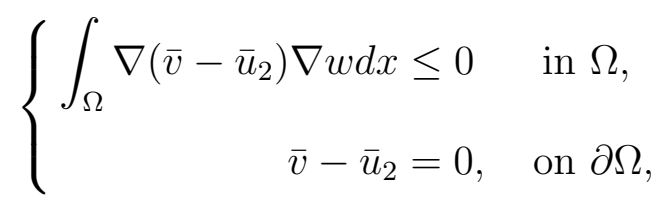

for every nonnegative $w \in H_{0}^{1}(\Omega)$. Now by applying weak maximum principle (Theorem 2.6.1), we can assert that

$$
\sup _{\Omega}\left(\bar{v}-\bar{u}_{2}\right) \leq \sup _{\partial \Omega}\left(\bar{v}-\bar{u}_{2}\right)^{+}=0
$$

which yields

$$
\bar{v} \leq \bar{u}_{2} \quad \text { a.e. in } \Omega \text {. }
$$


Using a similar argument we obtain that

$$
\inf _{\Omega}\left(\bar{v}-\underline{u}_{1}\right) \geq \inf _{\partial \Omega}\left(\bar{v}-\underline{u}_{1}\right)^{-}=0,
$$

and hence

$$
\underline{u}_{1} \leq \bar{v} \quad \text { a.e. in } \Omega \text {. }
$$

So from (4.9) and (4.10) we deduce that $\underline{u}_{1} \leq \bar{v} \leq \bar{u}_{2}$, i.e. $\bar{v} \in K$. To show that $\bar{v} \in \operatorname{Dom}(\psi)$, it remains to prove that $\bar{v} \in W^{2, p^{\prime}}(\Omega)$ as well. Indeed, recalling $A_{2}$, we have that

$$
\int_{\Omega}|g(x, \bar{u})|^{p^{\prime}} d x \leq \int_{\Omega} a(x)^{p^{\prime}}\left(1+|\bar{u}|^{p-1}\right)^{p^{\prime}} d x .
$$

But as $a(x) \in L^{\infty}(\Omega)$, by applying Hölder's inequality, we obtain

$$
\int_{\Omega}|g(x, \bar{u})|^{p^{\prime}} d x \leq\|a(x)\|_{L^{\infty}}^{p^{\prime}} \int_{\Omega}\left(1+|\bar{u}|^{p-1}\right)^{p^{\prime}} d x .
$$

Hence we get

$$
\begin{aligned}
\|g(x, \bar{u})\|_{L^{p^{\prime}}(\Omega)} & \leq\|a(x)\|_{L^{\infty}}\left\|1+|\bar{u}|^{p-1}\right\|_{L^{p^{\prime}}(\Omega)} \\
& \leq\|a(x)\|_{L^{\infty}}\left(\|1\|_{L^{p^{\prime}}(\Omega)}+\left\|\bar{u}^{p-1}\right\|_{L^{p^{\prime}}(\Omega)}\right) \\
& \leq C_{a}\left(1+\|u\|_{L^{p}}^{p-1}\right),
\end{aligned}
$$

for an appropriate constant $C_{a}$. As $\bar{u} \in L^{p}(\Omega)$, we can deduce that $g(x, \bar{u}) \in$ $L^{p^{\prime}}(\Omega)$. Now by applying $L^{p}$ elliptic regularity (Theorem 2.4.2), we get the desired result which is $\bar{v} \in W^{2, p^{\prime}}(\Omega)$ and hence $\bar{v} \in \operatorname{Dom}(\psi)$.

Finally, as $I[\cdot]$ has a critical point $\bar{u}$ and there exists $\bar{v} \in K \cap W^{2, p^{\prime}}(\Omega)$ satisfying (4.5), by Corollary 3.2.4 we can conclude that (4.2) has a solution. 


\section{Chapter 5}

\section{Supercritical Semilinear Elliptic Equations}

\section{$5.1 \quad$ Introduction}

To unveil another aspect of the new variational principle established in $[25,26]$ and its applications, in this chapter we use it as a main tool to prove that there is a strong solution for the following equation

$$
\left\{\begin{aligned}
-\Delta u+\lambda u & =\mu g(x, u)+b(x) & & \text { in } \Omega, \\
u & =0 & & \text { on } \partial \Omega .
\end{aligned}\right.
$$

where $\Omega$ is a bounded domain in $\mathbb{R}^{n}$ with smooth boundary, $\mu$ and $\lambda$ two parameters, $b \in L^{2}(\Omega)$, and $g: \Omega \times \mathbb{R} \rightarrow \mathbb{R}$ is a Carathéodory function.

A particular case of (5.1), which has been investigated extensively, is the following problem

$$
\left\{\begin{aligned}
-\Delta u+\lambda u & =\mu|u|^{p-2} u+b(x) & & \text { in } \Omega, \\
u & =0 & & \text { on } \partial \Omega
\end{aligned}\right.
$$


where $\mu>0$ and $b \in L^{2}(\Omega)$. Working on (5.2) with $\lambda=0$ and $\mu=1$, in [4] Bahri has proved that for $2<p<2^{*}$ where $2^{*}=2 n /(n-2)$ for $n \geq 3$ and $2^{*}=\infty$ for $n=2$, problem (5.2) admits infinitely many weak solutions for an open dense set of $b$ in $W^{-1,2}(\Omega)$. In [8] Barroso used a new version of Krasnoselskii's fixed point theorem for the sum of two operators given in [7] and proved the existence of a strong solution for (5.2) with $\lambda$ close to zero for any $p>2$ if $n=3$, for $2<p \leq(2 n-4) /(n-4)$ if $n>4$, and finally for $2<p \leq 3$ if $n=4$. In [5] and [35], with different methods, they also proved if $b \in L^{2}(\Omega)$ then problem (5.2) possesses an infinite number of distinct solutions for $2<p<p_{N}$ where $p_{N}$ is a constant less than the critical exponent $2^{*}$.

Here, as a consequence of our result, we prove that if $b \in L^{2}(\Omega), \lambda \geq 0$, and $\mu>0$ is small then problem (5.2) has a strong solution for any $p>2$ if $n \leq 4$, and for $2<p \leq(2 n-4) /(n-4)$ if $n>4$. We remark that the results in this chapter are original and they are already published in [9]. The importance of our result is that for this range of $p$, our problem includes supercritical nonlinearity as well. Furthermore, when $\partial \Omega$ is smooth enough, the new principle enables us to generalize this result by just increasing the regularity of $b(x)$. In other words, by setting $b \in W^{k, 2}(\Omega)$, we can show the existence of a strong solution $u \in W^{k+2,2}(\Omega)$ for $p>2$, if $n \leq 2(k+2)$, and for $2<p \leq 2(n-2(k+1)) /(n-2(k+2))$ if $2(k+2)<n<2(k+2)+4 /(k-1)$. It is worth noting that the smoothness of the solution is also determined in this result.

In addition, under appropriate assumptions on $g$, we generalize our result for the equation (5.1) and find a strong solution for the supercritical problem. Indeed, we shall prove the following result.

Theorem 5.1.1. Suppose $\Omega$ is a bounded domain in $\mathbb{R}^{n}$ and $\partial \Omega$ is of class $C^{1,1}$. Consider the problem (5.1), with $\lambda \geq 0$ and $\mu$ positive. Furthermore, assume $g: \Omega \times \mathbb{R} \rightarrow \mathbb{R}$ is a Carathéodory function which is increasing in $u$ and satisfies 
the following growth condition:

$$
|g(x, u)| \leq a|u|^{p-1}+c,
$$

where $a>0$ and $c>0$ are two constants. Let $b \in L^{d}(\Omega)$ for $d \geq 2$, and

$$
\begin{cases}2<p \leq \frac{(2 n-2 d)}{(n-2 d)} & \text { if } \quad n>2 d \\ 2<p & \text { if } \quad n \leq 2 d\end{cases}
$$

Then, problem (5.1) admits at least one solution in $W^{2, d}(\Omega)$, provided $\mu$ is small.

Note that as $g$ has critical growth and we do not have the compactness of the Sobolev embedding, the usual variational methods, such as mountain pass approach, do not apply here. However, as we see in this chapter the new variational approach will rectify this matter.

We start this chapter by the proof of Theorem 5.1.1. Following the steps of Corollary 3.2.4, we first find the critical point $\bar{u}$ of the new functional in a closed ball $K=\left\{u \in V,\|u\|_{W^{2, d}(\Omega)} \leq R\right\}$, where we can utilize the compact Sobolev embedding of Theorem 2.3.2. Having a solution $\bar{v}$ for the linear equation $A v=D \varphi(\bar{u})$ as a consequence of Theorem 2.4.4, we complete our proof by using $L^{p}$ estimates (Theorem 2.4.2) and Lemma 2.3.5 to show that this solution is in $K$. In this chapter, we also state the existence theorem for a smooth solution of the particular case (5.2). By choosing $K=\left\{u \in V,\|u\|_{W^{k+2,2}(\Omega)} \leq R\right\}$, and applying elliptic regularity (Theorem 2.4.1) and Lemma 2.3.3 in the second step of the proof, we proceed analogously to the proof of Theorem 5.1.1 to assert the desired result. 


\subsection{Main Result}

This section is devoted to the proof of Theorem 5.1.1. We discuss the special case (5.2) in the next sections.

Recalling Section 3.2, we briefly set up the associated version of the new functional $I[\cdot]$ to our problem first. Set $V$ to be the Banach space $H_{0}^{1}(\Omega) \cap L^{p}(\Omega)$ with the norm

$$
\|u\|_{V}:=\|u\|_{H_{0}^{1}(\Omega)}+\|u\|_{L^{p}(\Omega)} .
$$

and assume that $V^{*}$ is the topological dual of $V$. In this case we consider the closed convex subset of $V, K=\left\{u \in V ;\|u\|_{W^{2, d}(\Omega)} \leq R\right\}$, where $R$ is a positive real number to be chosen later.

For $g: \Omega \times \mathbb{R} \rightarrow \mathbb{R}$ satisfying the growth condition $|g(x, y)| \leq a|y|^{p-1}+c$, we define $G: \Omega \times \mathbb{R} \rightarrow \mathbb{R}$ by $G(x, y)=\int_{0}^{y} g(x, s) d s$. As seen in Section 3.2, in order to apply Corollary 3.2.4, we write equation (5.1) in the abstract form as

$$
A u=D \varphi(u)
$$

where the linear operator $A: \operatorname{Dom}(A) \subset V \rightarrow V^{*}$ is defined by $A v:=-\Delta v+\lambda v$, with $\lambda \geq 0$ for $v \in V$, and $\varphi: V \rightarrow \mathbb{R}$ is defined by

$$
\varphi(u):=\int_{\Omega} \mu G(x, u)+u b(x) d x .
$$

with $\mu>0$ and $b \in L^{d}(\Omega)$, for $d \geq 2$.

Considering $G^{*}: \Omega \times \mathbb{R} \rightarrow(-\infty,+\infty]$ to be the Fenchel dual of $G$, i.e. $G^{*}(x, s)=\sup _{t \in \mathbb{R}}\{t s-G(x, t)\}$, we now define $\psi$ as follows

$$
\psi(u):= \begin{cases}\int_{\Omega} \mu G^{*}\left(x, \frac{-\Delta u+\lambda u-b(x)}{\mu}\right) d x, & u \in K, \\ +\infty & u \notin K,\end{cases}
$$

with $\operatorname{Dom}(\psi)=\{u \in V ; \psi(u)<\infty\}$. Note that as $p>2$, for its conjugate $p^{\prime}=p /(p-1)$ we have $p^{\prime}<2 \leq d$. From the embedding $L^{d}(\Omega) \hookrightarrow L^{p^{\prime}}(\Omega)$, we can 
deduce that $A u=-\Delta u+\lambda u \in L^{p^{\prime}}(\Omega)$ for $u \in K$. Therefore, from Lemma 3.2.3 and the definition of $\psi$ it can be seen that $\psi(u)=\varphi^{*}(A u)$.

Finally, we set $I[u]=\psi(u)-\varphi(u)$. Thus, we have

$$
I[u]=\int_{\Omega} \mu G^{*}\left(x, \frac{-\Delta u+\lambda u-b(x)}{\mu}\right) d x-\int_{\Omega} \mu G(x, u)+u b(x) d x .
$$

for each $u \in K$.

In the remainder of this section before the proof of main results, we prove some preliminary results needed in either step of the proof.

In the first part of the proof, to show that $I[\cdot]$ has a critical point, we need to prove that $I[\cdot]$ is weakly lower semi-continuous.

Lemma 5.2.1. The functional $I: V \rightarrow(-\infty,+\infty]$, defined by

$$
I[u]=\psi(u)-\varphi(u)
$$

is weakly lower semi-continuous.

Proof. First, we claim that $\psi(\cdot)$ is weakly lower semi-continuous. Let $\left\{u_{n}\right\}$ be a sequence in $V$ that converges weakly to some $\bar{u} \in V$. If $\liminf _{n \rightarrow \infty} \psi\left(u_{n}\right)=\infty$, we have the result, so let us assume that $\liminf _{n \rightarrow \infty} \psi\left(u_{n}\right)<\infty$. By definition of $\psi(\cdot)$, we can deduce that $\left\{u_{n}\right\} \subset K$ and hence $\left\|u_{n}\right\|_{W^{2, d}(\Omega)} \leq R$, for all $n$. Then going if necessary to a subsequence, we can assume that $u_{n} \rightarrow \bar{u}$ weakly in $W^{2, d}(\Omega)$.

For $v \in L^{p}(\Omega)$, by definition of $\varphi^{*}\left(-\Delta u_{n}+\lambda u_{n}\right)$, we have that

$$
\psi\left(u_{n}\right)=\varphi^{*}\left(-\Delta u_{n}+\lambda u_{n}\right) \geq \int_{\Omega} v(x)\left(-\Delta u_{n}+\lambda u_{n}\right) d x-\varphi(v) .
$$

Let $d^{\prime}$ be the conjugate of $d$, i.e. $1 / d+1 / d^{\prime}=1$. So, $d \geq 2$ yields that $d^{\prime} \leq 2$. Considering the fact that $p>2 \geq d^{\prime}$ we can use the embedding $L^{p}(\Omega) \hookrightarrow L^{d^{\prime}}(\Omega)$, and obtain that $v \in L^{d^{\prime}}(\Omega)$. In addition, $-\Delta u_{n}+\lambda u_{n}$ is obviously in $L^{d}(\Omega)$. Hence, from the definition of weak convergence in $W^{2, d}(\Omega)$, we can deduce

$$
\liminf _{n \rightarrow \infty} \psi\left(u_{n}\right)=\liminf _{n \rightarrow \infty} \varphi^{*}\left(-\Delta u_{n}+\lambda u_{n}\right) \geq \int_{\Omega} v(x)(-\Delta \bar{u}+\lambda \bar{u}) d x-\varphi(v) .
$$


Taking sup over all $v \in L^{p}(\Omega)$ implies that

$$
\liminf _{n \rightarrow \infty} \psi\left(u_{n}\right)=\liminf _{n \rightarrow \infty} \varphi^{*}\left(-\Delta u_{n}+\lambda u_{n}\right) \geq \varphi^{*}(\Delta \bar{u}+\lambda \bar{u})=\psi(\bar{u}),
$$

which means that $\psi(\cdot)$ is weakly lower semi-continuous.

In addition, we prove that $\varphi(\cdot)$ is continuous. Recall that $u_{n} \rightarrow \bar{u}$ in $W^{2, d}(\Omega)$. Moreover, having $\partial \Omega \in C^{1,1}$, by Theorem (2.3.1) we can use the compact embeddings $W^{2, d}(\Omega) \hookrightarrow L^{q}(\Omega)$ for $1 \leq q<d n /(n-2 d)$ if $2 d<n$ and for $1 \leq q<\infty$ if $n \leq 2 d$, to conclude that up to a subsequence, $u_{n} \rightarrow \bar{u}$ strongly in $L^{p}(\Omega)$, for the range of $p$ in (5.4), and hence $u_{n} \rightarrow \bar{u}$ a.e. Therefore, from the continuity of $G(x, \cdot)$, which is a consequence of the continuity of $g(x, \cdot)$ as a Carathéodory function, we can deduce that $G\left(x, u_{n}\right) \rightarrow G(x, \bar{u})$ a.e., and so $\mu G\left(x, u_{n}\right)+b(x) u_{n} \rightarrow \mu G(x, \bar{u})+b(x) \bar{u}$, for a.e. $x \in \Omega$. Furthermore, considering the growth condition (5.3) and the fact that $G(x,$.$) is the primitive of g(x,$.$) we$ obtain

$$
G\left(x, u_{n}\right) \leq \frac{a}{p}\left|u_{n}\right|^{p}+c u_{n},
$$

which yields

$$
\mu G\left(x, u_{n}\right)+b(x) u_{n} \leq \frac{\mu a}{p}\left|u_{n}\right|^{p}+(b(x)+\mu c) u_{n}
$$

It can be easily seen that $\frac{\mu a}{p}\left|u_{n}\right|^{p}+(b(x)+\mu c) u_{n} \in L^{1}(\Omega)$. Indeed, by applying Hölder's inequality we find that

$$
\begin{aligned}
\int_{\Omega} \frac{\mu a}{p}|u|^{p}+(b(x)+\mu c) u d x & \leq \frac{\mu a}{p}\|u\|_{L^{p}(\Omega)}^{p}+\|b(x)+\mu c\|_{L^{d}(\Omega)}\|u\|_{L^{d^{\prime}}(\Omega)} \\
& \leq \frac{\mu a}{p}\|u\|_{L^{p}(\Omega)}^{p}+C\|b(x)+\mu c\|_{L^{d}(\Omega)}\|u\|_{L^{p}(\Omega)}<\infty,
\end{aligned}
$$

for an appropriate constant $C$. Therefore, by Lebesgue dominated converges theorem we find that

$$
\begin{aligned}
\lim _{n \rightarrow \infty} \int_{\Omega} \mu G\left(x, u_{n}\right)+u_{n} b(x) d x & =\int_{\Omega} \lim _{n \rightarrow \infty} \mu G\left(x, u_{n}\right)+u_{n} b(x) d x \\
& =\int_{\Omega} \mu G(x, \bar{u})+\bar{u} b(x) d x
\end{aligned}
$$


and hence $\varphi\left(u_{n}\right) \rightarrow \varphi(\bar{u})$, which means that $\varphi(\cdot)$ is continuous. Recall $u_{n} \rightarrow \bar{u}$. By definition we have

$$
\liminf _{n \rightarrow \infty} I\left[u_{n}\right]=\liminf _{n \rightarrow \infty}\left(\psi\left(u_{n}\right)-\varphi\left(u_{n}\right)\right),
$$

and since $\varphi(\cdot)$ is continuous, we obtain

$$
\liminf _{n \rightarrow \infty} I\left[u_{n}\right]=\liminf _{n \rightarrow \infty} \psi\left(u_{n}\right)-\lim _{n \rightarrow \infty} \varphi\left(u_{n}\right) \geq \psi(\bar{u})-\varphi(\bar{u})=I[\bar{u}],
$$

which means that $I[\cdot]$ is weakly lower semi-continuous.

In the second part of our proof, where we show that the linear equation $A v=D \varphi(\bar{u})$ admits a solution $\bar{v} \in K$, the following result is required.

Lemma 5.2.2. Let $d \geq 2$ and consider the linear equation

$$
\left\{\begin{aligned}
-\Delta v+\lambda v & =l(x), & & \text { in } \quad \Omega, \\
v & =0, & \text { on } \quad & \partial \Omega,
\end{aligned}\right.
$$

where $l \in L^{d}(\Omega)$ and $\lambda \geq 0$. Then for the weak solution $\bar{v}$ of (5.8) we have $\|\bar{v}\|_{L^{d}(\Omega)} \leq C_{d}\|l\|_{L^{d}(\Omega)}$ for some constant $C_{d}$ depending only on $d$.

Proof. Let $l_{n}$ be a sequence of smooth functions approaching $l$ in $L^{d}(\Omega)$ and assume that $v_{n}$ is a solution of

$$
\left\{\begin{array}{rlrl}
-\Delta v_{n}+\lambda v_{n} & =l_{n}(x), & & \text { in } \quad \Omega, \\
v_{n}=0, & & \text { on } \quad \partial \Omega .
\end{array}\right.
$$

As $v_{n}$ is a smooth solution of (5.9), we can multiply (5.9) by $\left|v_{n}\right|^{d-2} v_{n}$ and integrate to obtain

$$
\int_{\Omega}\left(-\Delta v_{n}+\lambda v_{n}\right)\left|v_{n}\right|^{d-2} v_{n} d x=\int_{\Omega} l_{n}\left|v_{n}\right|^{d-2} v_{n} d x,
$$

by integration by part and applying Hölder's inequality on the right hand side, we get

$$
\int_{\Omega}(d-1)\left|\nabla v_{n}\right|^{2}\left|v_{n}\right|^{d-2}+\lambda\left|v_{n}\right|^{d} \leq\left\|l_{n}\right\|_{L^{d}(\Omega)}\left\|v_{n}{ }^{d-1}\right\|_{L^{d^{\prime}}(\Omega)}
$$




$$
\leq\left\|l_{n}\right\|_{L^{d}(\Omega)}\left\|v_{n}\right\|_{L^{d}(\Omega)}^{d-1},
$$

where $d^{\prime}=d /(d-1)$ is the conjugate of $d$. Now by applying Pioncaré inequality we obtain

$$
\int_{\Omega}\left|\nabla v_{n}\right|^{2}\left|v_{n}\right|^{d-2} d x \geq\left.\left.\left(\frac{2}{d}\right)^{2} \int_{\Omega}|\nabla| v_{n}\right|^{\frac{d}{2}}\right|^{2} d x \geq C\left(\frac{2}{d}\right)^{2} \int_{\Omega}\left|v_{n}\right|^{d} d x=C\left(\frac{2}{d}\right)^{2}\left\|v_{n}\right\|_{L^{d}}^{d},
$$

where $C$ is a constant depending on $\Omega$. Hence it follows that

$$
\left\|v_{n}\right\|_{L^{d}(\Omega)} \leq C_{d}\left\|l_{n}\right\|_{L^{d}(\Omega)},
$$

for a constant $C_{d}$. The desired result follows by letting $n \rightarrow \infty$.

Now that we have all the required results, we can state the proof of the main theorem.

Proof of Theorem 5.1.1. Recall that

$$
K=\left\{u \in V ;\|u\|_{W^{2, d}(\Omega)} \leq R\right\}
$$

with $R$ a constant to be determined. To prove that (5.1) has a solution, using Corollary 3.2.4, first we must show that $I$ has a minimum in $K$, i.e.

$$
\exists \bar{u} \in K \quad \text { such that } \quad I[\bar{u}]=\inf _{u \in K} I[u] .
$$

It follows from the growth condition (5.3) that for a constant $C_{1}>0, G(x, u) \leq$ $\frac{a}{p}|u|^{p}+c u+C_{1}$. Then, for $u \in K$, that is $\|u\|_{W^{2, d}(\Omega)} \leq R$, by using Hölder's inequality we have

$$
\begin{aligned}
\varphi(u)=\int_{\Omega} \mu G(x, u)+u b(x) d x & \leq \int_{\Omega} \frac{\mu a}{p}|u|^{p}+(b(x)+\mu c) u+\mu C_{1} d x \\
& \leq \frac{\mu a}{p}\|u\|_{L^{p}(\Omega)}^{p}+\|b(x)+\mu c\|_{L^{d}(\Omega)}\|u\|_{L^{d^{\prime}}(\Omega)}+\mu C_{1}|\Omega|,
\end{aligned}
$$

where $d^{\prime}$ is the Sobolev conjugate of $d$, i.e. $1 / d+1 / d^{\prime}=1$. It follows from Theorem 2.3.2 that the embedding $W^{2, d}(\Omega) \hookrightarrow L^{q}(\Omega)$ is continuous for $1 \leq q<\infty$ if $n \leq 2 d$, and for $1 \leq q \leq n d /(n-2 d)$ if $n>2 d$. Recalling that by assumption 
$p>2$ if $n<2 d$ and $2<p \leq(2 n-2 d) /(n-2 d)$ if $n>2 d$, we can conclude the continuous embedding $W^{2, d} \hookrightarrow L^{p}(\Omega)$, for this range of $p$. In addition, $d \geq 2$ implies that $d^{\prime} \leq 2$ so clearly $d^{\prime}<n d /(n-2 d)$ for any $n$ and $d$, and hence the embedding $W^{2, d}(\Omega) \hookrightarrow L^{d^{\prime}}(\Omega)$ is also continuous. Thus we get

$$
\varphi(u) \leq \frac{\mu a}{p} C_{3}\|u\|_{W^{2, d}(\Omega)}^{p}+\|b(x)+\mu c\|_{L^{d}(\Omega)} C_{4}\|u\|_{W^{2, d}(\Omega)}+C_{2},
$$

for appropriate $C_{2}, C_{3}, C_{4}$. Since $\|u\|_{W^{2, d}(\Omega)} \leq R$ and $b \in L^{d}(\Omega)$ we obtain

$$
\varphi(u) \leq\left(C_{5} R^{p}+C_{4}\|b(x)+\mu c\|_{L^{d}(\Omega)} R+C_{2}\right)<\infty,
$$

for $C_{5}=\mu a C_{3} / p$. As a result, we see that

$$
I[u]=\varphi^{*}(A u)-\varphi(u) \geq \varphi^{*}(A u)-\left(C_{5} R^{p}+C_{4}\|b(x)+\mu c\|_{L^{d}(\Omega)} R+C_{2}\right) .
$$

from which one has $\inf _{u \in K} I[u]>-\infty$. We now consider a minimizing sequence in $K$ i.e.

$$
\left\{u_{n}\right\} \subset K ; \quad \text { such that } \quad I\left[u_{n}\right] \rightarrow \inf _{u \in K} I[u]:=m .
$$

Now we shall show that $I[\cdot]$ attains its minimum in $K$. Note that $\left\{u_{n}\right\} \subset K$ implies $\left\|u_{n}\right\|_{W^{2, d}(\Omega)} \leq R$, for all $n$, so we can deduce that $\left\{u_{n}\right\}$ is bounded in $W^{2, d}(\Omega)$. Going if necessary to a subsequence, and recalling that $W^{2, d}(\Omega)$ is reflexive, we can assume there exists $\bar{u} \in W^{2, d}(\Omega)$ that $u_{n} \rightarrow \bar{u}$ weakly in $W^{2, d}(\Omega)$. Moreover, as $K$ is convex and closed, and therefore weakly closed, $\bar{u}$ is also in $K$. From Lemma 5.2.1, we know that $I[\cdot]$ is weakly lower semi-continuous, so we can deduce that

$$
m \leq I[\bar{u}] \leq \liminf _{n \rightarrow \infty} I\left[u_{n}\right]=m,
$$

which means that $\bar{u} \in K$ is a minimizer of $I[\cdot]$.

Following Corollary 3.2.4, to show the existence of solution for (5.1), in the next step, we need to prove that there exists a $\bar{v} \in V \cap K$ satisfying

$$
\left\{\begin{aligned}
-\Delta v+\lambda v & =\mu g(x, \bar{u})+b(x), & & \text { in } \Omega, \\
v & =0, & & \text { on } \partial \Omega .
\end{aligned}\right.
$$


First we claim that (5.11) is satisfied by a $\bar{v} \in V$. Indeed, from the growth condition (5.3) it can be easily seen that

$$
\|g(x, \bar{u})\|_{L^{d}(\Omega)} \leq a\left\|\bar{u}^{p-1}\right\|_{L^{d}(\Omega)}+C_{6}
$$

for an appropriate constant $C_{6}$. Moreover, as shown in Lemma 2.3.5, for the range of $p$ in (5.4), we have $|\bar{u}|^{p-1} \in L^{d}(\Omega)$, and also

$$
\left\|\bar{u}^{p-1}\right\|_{L^{d}(\Omega)} \leq C_{p}\|\bar{u}\|_{W^{2, d}(\Omega)}^{p-1}
$$

so, by using triangle inequality, from (5.12) we obtain

$$
\begin{aligned}
\|\mu g(x, \bar{u})+b(x)\|_{L^{d}(\Omega)} & \leq\|\mu g(x, \bar{u})\|_{L^{d}(\Omega)}+\|b(x)\|_{L^{d}(\Omega)} \\
& \leq \mu a\left\|\bar{u}^{p-1}\right\|_{L^{d}(\Omega)}+\|b(x)\|_{L^{d}(\Omega)}+\mu C_{6} .
\end{aligned}
$$

Hence, we deduce that $\mu g(x, \bar{u})+b(x) \in L^{d}(\Omega)$ as we have $|\bar{u}|^{p-1}, b \in L^{d}(\Omega)$. Therefore, since $\partial \Omega$ is of class of $C^{1,1}$, by a standard result in the existence of strong solutions for the Dirichlet problem (Theorem 2.4.4), we can assert that problem (5.11) has a solution $\bar{v} \in W^{2, d}(\Omega)$. We can also apply the global elliptic regularity (Theorem 2.4.2) to obtain

$$
\|\bar{v}\|_{W^{2, d}(\Omega)} \leq C_{7}\left(\|\bar{v}\|_{L^{d}(\Omega)}+\|\mu g(x, \bar{u})+b(x)\|_{L^{d}(\Omega)}\right),
$$

for an appropriate constant $C_{7}$. Now to show $\|\bar{v}\|_{W^{2, d}(\Omega)} \leq R$, recall that from Lemma (5.2.2) we have

$$
\|\bar{v}\|_{L^{d}(\Omega)} \leq C_{d}\|\mu g(x, \bar{u})+b(x)\|_{L^{d}(\Omega)}
$$

which by applying (5.14) yields

$$
\|\bar{v}\|_{L^{d}(\Omega)} \leq C_{d}\left(\mu a\left\|\bar{u}^{p-1}\right\|_{L^{d}(\Omega)}+\|b(x)\|_{L^{d}(\Omega)}+\mu C_{6}\right) .
$$

Thus, by substituting (5.14) and (5.16) in (5.15) we have

$$
\|\bar{v}\|_{W^{2, d}(\Omega)} \leq C_{8}\left(\mu a\left\|\bar{u}^{p-1}\right\|_{L^{d}(\Omega)}+\|b(x)\|_{L^{d}(\Omega)}+\mu C_{6}\right),
$$


where $C_{8}=C_{7}\left(C_{d}+1\right)$. Therefore, by substituting (5.13) in (5.17), we obtain

$$
\|\bar{v}\|_{W^{2, d}(\Omega)} \leq C_{8}\left(\mu a C_{p}\|\bar{u}\|_{W^{2, d}(\Omega)}^{p-1}+\|b(x)\|_{L^{d}(\Omega)}+\mu C_{6}\right) .
$$

Now as $\bar{u} \in K$, i.e. $\|\bar{u}\|_{W^{2, d}(\Omega)} \leq R$, we get

$$
\|\bar{v}\|_{W^{2, d}(\Omega)} \leq C_{8}\left(\mu a C_{p} R^{p-1}+\|b(x)\|_{L^{d}(\Omega)}+\mu C_{6}\right) .
$$

Next, recall that $b \in L^{d}(\Omega)$, so we can choose $R$ such that $C_{8}\|b(x)\|_{L^{d}(\Omega)}<R$, then by fixing $R$, we can choose $\mu$ such that

$$
C_{8}\left(\mu a C_{p} R^{p-1}+\|b(x)\|_{L^{d}(\Omega)}+\mu C_{6}\right) \leq R
$$

As a result, for this specific value of $\mu$ we have

$$
\|\bar{v}\|_{W^{2, d}(\Omega)} \leq R
$$

which means that $\bar{v} \in K$.

Finally, as $I$ has a minimum $\bar{u}$ and there exists a $\bar{v} \in V \cap K$ satisfying (5.11), by Corollary 3.2.4 we can conclude that $\bar{u} \in W^{2, d}(\Omega)$ is a solution of (5.1).

\subsubsection{A Special Case}

In the special case when $d=2$, our assumption on $b$ coincides with the one assumed in $[4,5,35,8]$, however, as one can see in the following corollary, regarding the range of $p$, the comparable result in our case is still stronger than those achieved in the cited papers. We can restate our result for this case as follows.

Corollary 5.2.3. Suppose $\Omega$ is a bounded domain in $\mathbb{R}^{n}$ and $\partial \Omega$ is of class $C^{1,1}$. Consider the problem

$$
\left\{\begin{aligned}
-\Delta u+\lambda u & =\mu g(x, u)+b(x) & & \text { in } \Omega \\
u & =0 & & \text { on } \partial \Omega
\end{aligned}\right.
$$


with $\lambda \geq 0$ and $\mu$ positive. Furthermore, assume $g: \Omega \times \mathbb{R} \rightarrow \mathbb{R}$ is a Carátheodory function which is increasing and satisfies the following growth condition:

$$
|g(x, u)| \leq a|u|^{p-1}+c
$$

where $a>0$ and $c>0$ are two constants. Let $b \in L^{2}(\Omega)$, and

$$
\begin{cases}2<p \leq \frac{(2 n-4)}{(n-4)} & \text { if } \quad n>4 \\ 2<p & \text { if } \quad n \leq 4\end{cases}
$$

Then, problem (5.18) admits at least one solution provided $\mu$ is small.

\subsubsection{Smooth Solutions}

As mentioned in the introduction, when it comes to problem (5.2), in addition to the existence of a solution for the problem with supercritical nonlinearity, by choosing $b$ to be smooth enough and applying Theorem 2.4.1, we can also determine the smoothness of the solution. Therefore we dedicate the rest of this section to the proof of this interesting result, which we can state as follows.

Theorem 5.2.4. Suppose $\Omega$ is a bounded domain in $\mathbb{R}^{n}$ and $\partial \Omega$ is of class of $C^{k+2}$. Consider following problem

$$
\left\{\begin{aligned}
-\Delta u+\lambda u & =\mu|u|^{p-2} u+b(x) & & \text { in } \Omega, \\
u & =0 & & \text { on } \partial \Omega,
\end{aligned}\right.
$$

where $\lambda \geq 0$ and $\mu>0$ are two real parameters. Assume that $b \in W^{k, 2}(\Omega)$, and either

$$
1<k \leq p-1, \text { and } \begin{cases}2<p \leq \frac{2(n-2(k+1))}{n-2(k+2)} & \text { if } \quad 2(k+2)<n \leq 2(k+2)+\frac{4}{k-1}, \\ 2<p & \text { if } \quad n \leq 2(k+2),\end{cases}
$$


or

$$
k=0,1, \text { and } \begin{cases}2<p \leq \frac{2(n-2(k+1))}{n-2(k+2)} & \text { if } \quad 2(k+2)<n, \\ 2<p & \text { if } \quad n \leq 2(k+2) .\end{cases}
$$

Then, problem (5.20) admits at least one solution in $W^{k+2,2}(\Omega)$ for $\mu$ sufficiently small.

Before the proof, first we set up $I[\cdot]$ for this special case for reader's convenience. Setting $g(x, y)=|y|^{p-2} y$, we define

$$
G(x, y)=\int_{0}^{y}|s|^{p-2} s d s=\frac{1}{p}|y|^{p} .
$$

Hence, we can define $\varphi: V \rightarrow \mathbb{R}$ to be

$$
\varphi(u):=\int_{\Omega} \frac{\mu}{p}|u|^{p}+u b(x) d x,
$$

so by definition $\varphi(\cdot)$ is Gâteaux differentiable, convex and lower semi-continuous. Recalling Lemma 3.2.3, by an easy calculation we find the Fenchel dual $\varphi^{*}(h)$ : $V^{*} \rightarrow \mathbb{R}$ of $\varphi$ by

$$
\varphi^{*}(h)=\frac{\mu^{1-p^{\prime}}}{p^{\prime}} \int_{\Omega}|h-b(x)|^{p^{\prime}} d x, \quad \text { for } h \in L^{p}(\Omega),
$$

where as before $p^{\prime}$ is the conjugate of $p$. Looking for smooth solutions, we set $K=\left\{u \in V ;\|u\|_{W^{k+2,2}(\Omega)} \leq R\right\}$, and define $\psi$ as below

$$
\psi(u):= \begin{cases}\frac{\mu^{1-p^{\prime}}}{p^{\prime}} \int_{\Omega}|-\Delta u+\lambda u-b(x)|^{p^{\prime}} d x & u \in K, \\ +\infty & u \notin K,\end{cases}
$$

with $\operatorname{Dom}(\psi)=\{u \in V ; \psi(u)<\infty\}$. Now, we can define the new energy functional $I[\cdot]$ on $K$ as follows

$$
I[u]=\psi(u)-\varphi(u)=\frac{\mu^{1-p^{\prime}}}{p^{\prime}} \int_{\Omega}|-\Delta u+\lambda u-b(x)|^{p^{\prime}} d x-\frac{\mu}{p} \int_{\Omega}|u|^{p}+u b(x) d x .
$$

Note that this $I[\cdot]$ is a special case of (5.7). In spite of the different definition of the set $K$ for this case, it can be proved in much the same way as in Lemma 5.2.1 that (5.24) is weakly lower semi-continuous as well. 
We shall now prove Theorem 5.2.4 again by applying Corollary 3.2.4. It is worth noting that this proof takes the same steps as in proof of Theorem 5.1.1, however for the convenience of the reader we bring the proof here.

Proof of Theorem 5.2.4. Applying Corollary 3.2.4, we start the proof by showing that $I$ has a nontrivial critical point, which is a minimizer, i.e.

$$
\exists \bar{u} \in V \quad \text { such that } \quad I[\bar{u}]=\inf _{u \in V} I[u] .
$$

Using Hölder's inequality on $\varphi$, we obtain

$$
\begin{aligned}
\varphi(u) & =\int_{\Omega} \frac{\mu}{p}|u|^{p}+u b(x) d x \\
& \leq \frac{\mu}{p}\|u\|_{L^{p}(\Omega)}^{p}+\|b(x)\|_{L^{2}(\Omega)}\|u\|_{L^{2}(\Omega)} .
\end{aligned}
$$

As $\partial \Omega \in C^{k+2}$, we can consider the following compact embeddings, which we have from Theorem 2.3.2,

$$
\begin{array}{ll}
\text { if } \quad 2(k+2) \geq n, & W^{k+2,2}(\Omega) \hookrightarrow L^{q}(\Omega), \quad \text { for } \quad 1 \leq q<\infty, \\
\text { if } \quad 2(k+2)<n, & W^{k+2,2}(\Omega) \hookrightarrow L^{q}(\Omega), \quad \text { for } \quad 1 \leq q<\frac{2 n}{n-2(k+2)},
\end{array}
$$

so it can be deduced by assumptions (5.21) and (5.22) that $W^{k+2,2}(\Omega) \hookrightarrow L^{p}(\Omega)$ for the range of $p$ which we have in our problem. We also know that $W^{k, 2}(\Omega) \hookrightarrow$ $L^{2}(\Omega)$. As a result, from (5.25) we get

$$
\varphi(u) \leq \frac{\mu}{p} C_{1}^{\prime}\|u\|_{W^{k+2,2}}^{p}+C_{2}^{\prime}\|b(x)\|_{W^{k, 2}(\Omega)}\|u\|_{W^{k+2,2}(\Omega)}
$$

where $C_{1}^{\prime}$, and $C_{2}^{\prime}$ are constant. Recalling that $\|u\|_{W^{k+2,2}(\Omega)} \leq R$, and $b \in W^{k, 2}(\Omega)$, we thus have

$$
\varphi(u) \leq\left(C_{1}^{\prime} \frac{\mu}{p} R^{p}+C_{2}^{\prime}\|b(x)\|_{W^{k, 2}(\Omega)} R\right)<\infty .
$$

Hence,

$$
I[u]=\varphi^{*}(A u)-\varphi(u) \geq \varphi^{*}(A u)-\left(C_{1}^{\prime} \frac{\mu}{p} R^{p}+C_{2}^{\prime}\|b(x)\|_{W^{k, 2}(\Omega)} R\right) .
$$


Thus, $\inf _{u \in K} I[u]>-\infty$ and we can consider a minimizing sequence in $K$, i.e.

$$
\left\{u_{n}\right\} \subset K ; \quad \text { such that } \quad I\left[u_{n}\right] \rightarrow \inf _{u \in K} I[u]:=m .
$$

Now by an argument similar to the one in the proof of Theorem 5.1.1, as a result of the boundedness of $\left\{u_{n}\right\} \subset K$ in $W^{k+2,2}(\Omega)$, that is $\left\|u_{n}\right\|_{W^{k+2,2}(\Omega)} \leq R$, we can consider a $\bar{u} \in W^{k+2,2}(\Omega)$ that up to a subsequence $u_{n} \rightarrow \bar{u}$ weakly in $W^{k+2,2}(\Omega)$, and so $\bar{u} \in K$. Then, recalling the lower semi continuity of $I[\cdot]$, deduced from Lemma (5.2.1), again we obtain

$$
m \leq I[\bar{u}] \leq \liminf _{n \rightarrow \infty} I\left[u_{n}\right]=m
$$

which means that $\bar{u} \in K$ is a minimizer of $I[\cdot]$.

Continuing as in the proof of Theorem 5.1.1, to show the existence of a solution for (5.20), in the next step, we need to prove that there exists a $\bar{v} \in V \cap K$ satisfying

$$
\left\{\begin{aligned}
-\Delta v+\lambda v & =\mu|\bar{u}|^{p-2} \bar{u}+b(x), & & \text { in } \Omega, \\
v & =0, & & \text { on } \partial \Omega,
\end{aligned}\right.
$$

First, we show that a $\bar{v} \in V$ satisfies this linear equation. Consider the EulerLagrange energy functional for $(5.27)$ on $H_{0}^{1}(\Omega)$,

$$
E(v)=\frac{1}{2} \int_{\Omega}|\nabla v|^{2}+\lambda|v|^{2} d x-\int_{\Omega}|\bar{u}|^{p-2} \bar{u} v+v b(x) d x,
$$

which is well-defined as $v \in H_{0}^{1}(\Omega), b \in W^{k, 2}(\Omega)$, and $\bar{u} \in W^{k+2,2}(\Omega)$ yields $\bar{u} \in L^{2(p-1)}(\Omega)$ for the range of $p$ which is assumed in (5.21) for this problem. Set

$$
E(\bar{v})=\inf _{v \in V} E(v),
$$

then, obviously $\bar{v}$ is a critical point of $E(v)$ and satisfies (5.27).

Furthermore, we must show $\bar{v} \in K$, i.e. $\|\bar{v}\|_{W^{k+2,2}(\Omega)} \leq R$. First, note that form triangle inequality we obtain

$$
\left\|\mu|\bar{u}|^{p-1}+b(x)\right\|_{W^{k, 2}(\Omega)} \leq \mu\left\|\bar{u}^{p-1}\right\|_{W^{k, 2}(\Omega)}+\|b(x)\|_{W^{k, 2}(\Omega)}
$$


As shown in Lemma 2.3.3, $|\bar{u}|^{p-1} \in W^{k, 2}(\Omega)$ and we have

$$
\left\|\bar{u}^{p-1}\right\|_{W^{k, 2}(\Omega)} \leq C_{p}\|\bar{u}\|_{W^{k+2,2}(\Omega)}^{p-1} .
$$

Also, by assumption we have $b \in W^{k, 2}(\Omega)$. Hence, from (5.28) we can deduce that $\mu|\bar{u}|^{p-2} \bar{u}+b(x) \in W^{k, 2}(\Omega)$. Beside this, we also have that $\bar{v} \in H_{0}^{1}(\Omega)$ is a solution of (5.27), and $\partial \Omega$ is of class of $C^{k+2}$, so we can apply the global regularity theory (Theorem 2.4.1) to find

$$
\|\bar{v}\|_{W^{k+2,2}(\Omega)} \leq C_{3}^{\prime}\left(\|\bar{v}\|_{L^{2}(\Omega)}+\left\|\mu|\bar{u}|^{p-1}+b(x)\right\|_{W^{k, 2}(\Omega)}\right),
$$

where $C_{3}^{\prime}$ is an appropriate constant. Moreover, from Lemma 5.2.2 and the embedding $W^{k, 2}(\Omega) \hookrightarrow L^{2}(\Omega)$ we have

$$
\begin{aligned}
\|\bar{v}\|_{L^{2}(\Omega)} & \leq C_{d}\left(\mu\left\|\bar{u}^{p-1}\right\|_{L^{2}(\Omega)}+\|b(x)\|_{L^{2}(\Omega)}\right) \\
& \leq C_{4}^{\prime}\left(\mu\left\|\bar{u}^{p-1}\right\|_{W^{k, 2}(\Omega)}+\|b(x)\|_{W^{k, 2}(\Omega)}\right),
\end{aligned}
$$

for an appropriate constant $C_{4}^{\prime}$. Hence, by applying (5.28) and (5.31) in (5.30) we have

$$
\|\bar{v}\|_{W^{k+2,2}(\Omega)} \leq C_{5}^{\prime}\left(\mu\left\|\bar{u}^{p-1}\right\|_{W^{k, 2}(\Omega)}+\|b(x)\|_{W^{k, 2}(\Omega)}\right),
$$

where $C_{5}^{\prime}=C_{3}^{\prime}\left(C_{4}^{\prime}+1\right)$. Consequently, by substituting (5.29) in (5.32) we get

$$
\|\bar{v}\|_{W^{k+2,2}(\Omega)} \leq C_{5}^{\prime}\left(C_{p} \mu\|\bar{u}\|_{W^{k+2,2}(\Omega)}^{p-1}+\|b(x)\|_{W^{k, 2}(\Omega)}\right) .
$$

Now as $\bar{u} \in K$, i.e., $\|\bar{u}\|_{W^{k+2,2}(\Omega)} \leq R$, we obtain

$$
\|\bar{v}\|_{W^{k+2,2}(\Omega)} \leq C_{5}^{\prime}\left(C_{p} \mu R^{p-1}+\|b(x)\|_{W^{k, 2}(\Omega)}\right) .
$$

Furthermore, as $b \in W^{k, 2}(\Omega)$, we can choose $R$ such that $C_{5}^{\prime}\|b(x)\|_{W^{k, 2}(\Omega)}<R$, then by fixing $R$, we can find $\mu$ such that

$$
C_{5}^{\prime}\left(C_{p} \mu R^{p-1}+\|b(x)\|_{W^{k, 2}(\Omega)}\right) \leq R
$$

Thus, for this specific value of $\mu$, we have

$$
\|\bar{v}\|_{W^{k+2,2}(\Omega)} \leq R
$$


which means that $\bar{v} \in K$.

As a result, since $I$ has a minimum $\bar{u}$ on $K$ and there exists a $\bar{v} \in V \cap K$ satisfying (5.27), by Corollary 3.2.4 we deduce that $\bar{u} \in W^{k+2,2}(\Omega)$ is a solution for $(5.20)$.

Remark 5.2.5. Note that in Theorem 5.2.4, we are mostly concerned with the regularity of the solution. Otherwise, the existence of a solution can be proved by applying Theorem 5.1.1 too. Indeed, from the embedding $W^{k, 2}(\Omega) \hookrightarrow L^{d}(\Omega)$, for $1 \leq d<\infty$ when $n \leq 2 k$, and for $1 \leq d \leq 2 n /(n-2 k)$ when $n>2 k$, we have that $b(x) \in L^{d}(\Omega)$. Hence, Theorem 5.1 .1 implies the existence of a solution for

$$
\begin{cases}2<p \leq \frac{2(n-2(k+1))}{n-2(k+2)} & \text { if } \quad 2(k+2)<n \\ 2<p & \text { if } \quad n \leq 2(k+2)\end{cases}
$$

This solution belongs to $W^{2, d}(\Omega)$ where $1 \leq d<\infty$ if $n \leq 2 k$, and $1 \leq d \leq$ $2 n /(n-2 k)$ if $n>2 k$, whereas, the solution that is found by Theorem 5.2.4 is more regular and belongs to $W^{k+2,2}(\Omega)$. 


\section{Bibliography}

[1] R. A. Adams and J.J.F. Fournier, Sobolev Spaces, Pure and Applied Mathematics. Elsevier Science, (2003).

[2] K. Ako, On the Dirichlet problem for quasi-linear elliptic differential equations of the second order, J. Math. Soc. Japan 13 (1961), 45-62.

[3] I. Ali and A. Castro, Positive solution for a semilinear elliptic problem with critical exponent, Nonlinear Anal. Theory Methods. Appl. 27 (1996), no.3, 327-338.

[4] A. Bahri, Topological results on a certain class of functional and application, J. Func. Anal. 41 (1981), 397-427.

[5] A. Bahri and H. Berestycki, A perturbation method in critical point theory and applications, Trans. Amer. Math. Soc. 267 (1981), 1-32.

[6] V. Barbu and T. Precupanu, Convexity and Optimization in Banach Spaces, Springer Netherlands, Dordrecht, (2012).

[7] C. S. Barroso, Krasnoselskiis fixed point theorem for weakly continuous maps, Non-linear Anal. 55 (2003), 25-31.

[8] C. S. Barroso, Semilinear elliptic equations and fixed points, Proc. Amer. Math. Soc. 133 (2005), 745-749. 
[9] M. Basiri and A. Moameni, Solutions of supercritical semilinear nonhomogeneous elliptic problems, Nonlinear Anal. 165 (2017), 121-142.

[10] H. Brezis and L. Nirenberg, Positive solutions of nonlinear elliptic equations involving critical sobolev exponent, Comm. Pure Appl. Math. 36 (1983), 437447.

[11] D. Cao and H. Zhou, Multiple positive solutions of nonhomogeneous semilinear elliptic equations in $\mathbb{R}^{n}$, Proc. Roy. Soc. Edinburgh: Sect. A 126 (1996), 443-463.

[12] F.S. Cîrstea V. Radulescu, Multiple solutions of degenerate perturbed elliptic problems involving a subcritical sobolev exponent, Topol. Methods Nonlinear Anal. 15 (2000), 285-300.

[13] C. Cowan and A. Moameni, A new variational principle, convexity and supercritical Neumann problems, To appear in Trans. Amer. Math. Soc. (2017), pp. 34 .

[14] Ph. Clèment and G. Sweers, Getting a solution between sub- and supersolutions without monotone iteration, An International Journal of Mathematics 19 (1987), 189-194.

[15] E. N. Dancer and G. Sweers, On the existence of a maximal weak solution for a semilinear elliptic equation, Differential Integral Equations 2 (1989), no.4, 533-540.

[16] D.G. De Figueiredo and B. Ruf, Existence and non-existence of radial solutions for elliptic equations with critical exponent in $\mathbb{R}^{2}$, Comm Pure Appl. Math. 48 (1995), 639-655. 
[17] J. Deuell and P. Hess, A criterion for the existence of solutions of nonlinear elliptic boundary value problems, Proc. Royal Soc. Edinburgh Sect. A 74 (1976), 49-54.

[18] J. Dolbeault and I. Flores, Geometry of phase space and solutions of semilinear elliptic equations in a Ball, Trans. Amer. Math. Soc. 359 (2007), no.9, 4073-4087.

[19] I. Ekeland, Convexity Methods in Hamiltonian Mechanics, Springer-Verlag, Berlin, Heidelberg, New-York (1990).

[20] I. Ekeland and R. Temam, Convex analysis and variational problems, American Elsevier Publishing Co., Inc., New York, (1976).

[21] L. C. Evans, Partial Differential Equations, AMS, (1998).

[22] D. Gilbarg D. and N.S. Trudinger, Elliptic partial differential equations of second order, Classics in Mathematics. Springer-Verlag, Berlin, (2001).

[23] J.L. Kazdan and F.W. Warner, Remarks on some quasilinear elliptic equations, Comm. Pure Appl. Math. 28 (1975), no.5, 576-597.

[24] P.L. Lions, On the existence of positive solutions of semilinear elliptic equations, SIAM Review 24 (1982), 441-467.

[25] A. Moameni, Non-convex self-dual Lagrangians: new variational principles of symmetric boundary value problems, J. Funct. Anal. 260 (2011), no. 9, 2674-2715.

[26] A. Moameni, New variational principles of symmetric boundary value problems, J. Convex Anal. 24 (2017), no. 2, 365-381.

[27] M. Montenegro and A.C. Ponce, The sub-supersolution method for weak solutions, Amer. Math. Soc. 136 (2008), no.7, 2429-2438. 
[28] D. Passaseo, Nonexistence results for elliptic problems with supercritical nonlinearity in nontrivial domains, J. Funct. Anal. 114 (1992), 97-105.

[29] D. Passaseo, Multiplicity of nodal solutions for elliptic equations with supercritical exponent in contractible domains, Topol. Methods Nonlinear Anal. 8 (1996), 245-262.

[30] S.I. Pohožaev, On the eigenfunctions of the equation $\Delta u+\lambda f(u)=0$, Soviet. Math. Dokl. 6 (1965), 1408-1411.

[31] P. H. Rabinowitz, Minimax methods in critical point theory with applications to differential equations, CBMS Regional Conference Series Math. 65, Amer. Math. Soc., Providence (1986).

[32] O. Rey, On a variational problem with lack of compactness: The effect of small holes in the domain, C.R. Acad. Sci. Paris Sér. I Math. 308 (1989), no. $12,349-352$.

[33] H.L. Royden and P. Fitzpatrick, Real Analysis, Prentice Hall,(2010).

[34] J. Smoller, Shock waves and reaction-diffusion equations, Springer-Verlag, Berlin, (1983).

[35] M. Struwe, Infinitely many critical points for functionals which are not even and applications to super linear boundary value problems, Manuscripta mathematica 32 (1980), 335-364.

[36] M. Struwe, Variational Methods, Applications to Nonlinear Partial Differential Equations and Hamiltonian Systems, Springer, Berlin,(1990)

[37] A. Szulkin, Minimax principles for lower semicontinuous functions and applications to nonlinear boundary value problems. Ann. Inst. H. Poincaré Anal. Non Linéaire 3 (1986), no. 2, 77-109. 
[38] M.M. Vainberg, Variational methods for the study of non-linear operators. Holden-Day, SanFrancisco, (1964).

[39] M. Willem, Minimax Theorems, Birkhäuser, Boston, (1997).

[40] J. Zhiren, Multiple solutions for a class of semilinear elliptic equations, Proc. Amer. Math. Soc. 125 (1997), 3659-3667. 